\title{
Efficient simulation of one-dimensional two-phase flow with a high-order $h$-adaptive space-time Discontinuous Galerkin method
}

\author{
J.S.B. van Zwieten ${ }^{\mathrm{a}, *}$, B. Sanderse ${ }^{c, d}$, M.H.W. Hendrix ${ }^{\mathrm{b}}$, C. Vuik ${ }^{\mathrm{a}}$, R.A.W.M. Henkes ${ }^{\mathrm{c}, \mathrm{b}}$ \\ a Delft Institute of Applied Mathematics, TU Delft, Delft, Netherlands \\ ${ }^{\mathrm{b}}$ Department of Process and Energy, TU Delft, Delft, Netherlands \\ ' Shell Technology Centre Amsterdam, Amsterdam, Netherlands \\ d Centrum Wiskunde \& Informatica (CWI), Amsterdam, The Netherlands
}

\section{A R T I C L E I N F O}

\section{Article history:}

Received 30 May 2016

Revised 31 March 2017

Accepted 14 June 2017

Available online 15 June 2017

\section{Keywords:}

Two-fluid model

Discontinuous Galerkin method

$h$-adaptive

\begin{abstract}
A B S T R A C T
One-dimensional models for multiphase flow in pipelines are commonly discretised using first-order Finite Volume (FV) schemes, often combined with implicit time-integration methods. While robust, these methods introduce much numerical diffusion depending on the number of grid points. In this paper we propose a high-order, space-time Discontinuous Galerkin (DG) Finite Element method with $h$-adaptivity to improve the efficiency of one-dimensional multiphase flow simulations. For smooth initial boundary value problems we show that the DG method converges with the theoretical rate and that the growth rate and phase shift of small, harmonic perturbations exhibit superconvergence. We employ two techniques to accurately and efficiently represent discontinuities. Firstly artificial diffusion in the neighbourhood of a discontinuity suppresses spurious oscillations. Secondly local mesh refinement allows for a sharper representation of the discontinuity while keeping the amount of work required to obtain a solution relatively low. The proposed DG method is shown to be superior to FV.
\end{abstract}

(c) 2017 Published by Elsevier Ltd.

\section{Introduction}

Multiphase flow plays an important role in many industrial applications, such as in the petroleum and nuclear industry. In the petroleum industry a typical example of multiphase flow is the transport of oil and gas through long multiphase pipeline systems. For the design and optimization of such systems it is important to accurately predict the pressure and flow rate of both oil and gas along the pipeline as a function of time. An important example is the prediction of slug flow, which has a large influence on the sizing of receiving facilities at the outlet of the pipeline such as slug catchers or separators. A slug is a pocket of liquid that fully covers the pipe cross sectional area and that moves with relatively high velocity along the pipeline. Some slugs are initiated due to a flow instability at the gas/liquid interface of stratified flow in the pipeline, which marks the transition from stratified flow to hydrodynamic slug flow. The motion of these slugs, and of oil and gas in general, is governed by partial differential equations describing conservation of mass, momentum and energy. However, for oil and gas pipelines the numerical solution of these equations in three di-

\footnotetext{
* Corresponding author.

E-mail addresses: joostvanzwieten@gmail.com, j.s.b.vanzwieten@tudelft.nl (J.S.B. van Zwieten).
}

mensions is prohibitively expensive due to the multi-scale nature of the problem: the pipeline length can be of the order of $100 \mathrm{~km}$, whereas the size of oil droplets or gas bubbles can be of the order of millimetres. In order to obtain a computationally tractable model which retains the most important physical effects, averaging techniques are typically applied to the governing equations, leading to a one-dimensional model. The one-dimensional two-fluid model [1,2] is the most commonly used model to simulate twophase flow in pipelines or channels. It is capable of describing the transition from stratified flow to slug flow [3]. As such, the twofluid model is a slug-capturing model in which slugs are a result of growing hydrodynamic instabilities.

Numerical solutions to the two-fluid model equations are in general obtained by finite difference methods or finite volume methods, both in commercial codes such as OLGA [4] and LedaFlow as well as in academic research codes [3,5-8]. These finite difference and finite volume methods are almost exclusively first order in space and time. For example, the slug capturing code TRIOMPH from [3] uses a finite volume method on a staggered grid, being first order accurate both in space and time. A main reason for the use of first order schemes is related to the ill-posedness of the basic two-fluid model (when surface tension or hydrostatic pressure variation are not taken into account) and its non-conservative nature. These properties make the application of high-order meth- 
ods as developed for single-phase flow (such as Essentially NonOscillatory (ENO) schemes) non-trivial. The artificial diffusion introduced by first order methods effectively regularizes the differential equations through damping non-physical instabilities associated with ill-posedness [9]. However, a major disadvantage of first order methods is that any physical instabilities will also be damped due to excessive numerical diffusion [6]. As a result, very fine meshes are required (see e.g. [3]); Bonizzi and Issa [10] recommend that the grid size should be less than half of the diameter of the pipe to capture the natural growth of disturbances. For practical pipeline simulations this is computationally far too expensive.

A few studies on the use of high-order methods for the numerical solution of the two-fluid model have been performed. Holmås et al. [11] use a pseudo-spectral Fourier method to solve the twofluid model and indicate a gain in computational time of several orders of magnitude with respect to classical finite difference schemes; especially the first order upwind method has excessive numerical diffusion. Fullmer et al. [9] show improved accuracy of a second order method over a first order method, although the second order method leads to non-monotone results. In all cases, these high-order upwind schemes can have unfavourable stability properties [6], giving a numerical growth rate which is quite different from the physical growth rate of instabilities. Consequently, high-order methods are not yet commonly applied for solving the two-fluid model equations.

The purpose of this paper is to present an efficient highorder numerical method that can simulate stratified and slug flow by solving the compressible two-fluid model. To overcome the common issues associated with high-order methods we propose an $h$-adaptive space-time Discontinuous-Galerkin Finite Element Method (DGFEM) scheme. This method allows a mesh to be refined locally ( $h$-refinement). In smooth regions of the flow a coarse mesh is used, while a fine mesh is used to resolve the physics around sharp gradients, such as near a slug front or tail, or when the flow becomes locally single phase. This is believed to lead to a more efficient numerical method compared to classical low-order finite difference or finite volume methods on fixed grids. The scheme can be extended to include $p$-coarsening near discontinuities.

Several quite different adaptive space-time DG methods with adaptive refinement have been described. The tent-pitcher algorithm $[12,13]$ creates a partial ordering of unstructured elements in space-time such that a discrete system can be solved on each element solely based on boundary data from lower elements in the partial ordering. Multiple elements can be solved for simultaneously if they are independent of each other. Since all characteristics should exit an element face in the same direction, this would yield very flat elements (in time) if the eigenvalues of the system have a very large positive and negative component.

Another technique proposed by Gassner et al. [14] involves a set of elements that are unstructured in space and extruded in time, where the time length of an element is variable. The flux contribution to an element is applied separately from the volume contribution after the volume contributions of all neighbouring elements have been computed. The scheme allows local $h$ - and $p$-adaptation. Since this method is essentially explicit, the time length restriction is severe for problems with very large characteristic speeds.

For a multidimensional multiphase flow application Sollie et al. [15] use a structured space-time base mesh subdivided in timeslabs, a sequence of sets of elements with the same time interval. A discrete system is solved per time-slab using an explicit integration scheme for pseudo time. Coarse elements in which there is an interface, described by a level set on the coarse mesh, are subdivided, allowing locally unstructured elements, such that the interface matches element boundaries. This front tracking scheme requires several iterations to recompute the refinement as the level set depends on the flow field and vice versa. In one-dimensional multiphase flow applications this scheme requires, in absence of a level set, a non-trivial mechanism to locate jumps in the liquid hold-up within an element.

Fidkowski and Luo [16] describe an adjoint based adaptive space-time DG scheme for the compressible Navier-Stokes equations. The space-time mesh is the tensor product of an unstructured spatial mesh and time-slabs. Both the spatial mesh and the set of time-slabs can be refined locally, maintaining the tensor product structure of the space-time mesh. The refinement decision is based on the solution of an adjoint problem and requires storing the solution on all time-slabs, which is infeasible for long running simulations.

In this paper we use a structured coarse space-time mesh, divided into time-slabs, and allow repeated, structured refinements in space and time of individual elements. Per element the decision to refine is based on a smoothness indicator. Spurious oscillations in the neighbourhood of discontinuities are suppressed by adding artificial viscosity to the model $[17,18]$.

The outline of this paper is as follows. In Section 2 we recall the governing equations of the compressible two-fluid model, and introduce a new term associated with the hydrostatic pressure variation which is generally neglected in the literature. In Section 3 the new $h$-adaptive DGFEM discretisation for the compressible two-fluid model is introduced. In Section 4 a second order Finite Volume discretisation of the same compressible twofluid model is given, which will be used to assess the performance of the new DGFEM. In Section 5 we analyse the stability of the two-fluid model and the DGFEM discretisation. Section 6 shows the results for two representative test cases.

\section{Governing equations of the compressible two-fluid model}

We employ two different one-dimensional models for the simulation of two-phase flow. We label the two phases with $G$ for gas and $\mathrm{L}$ for liquid, but the following also applies to a lighter liquid and a heavier liquid. For both models we assume that at least one phase is compressible.

\subsection{Two-fluid model}

The first, and most general, of the two models is a two-fluid model for stratified flow in a horizontal, round pipe. Each phase is represented by a mass and momentum balance equation, respectively given by

$\partial_{t}\left(A_{\beta} \rho_{\beta}\right)+\partial_{s}\left(A_{\beta} \rho_{\beta} u_{\beta}\right)=0$,

and

$$
\begin{gathered}
\partial_{t}\left(A_{\beta} \rho_{\beta} u_{\beta}\right)+\partial_{s}\left(A_{\beta} \rho_{\beta} u_{\beta}^{2}+A_{\beta} p_{\mathrm{av}, \beta}-A_{\beta} p_{\text {int }}\right) \\
+A_{\beta} \partial_{s} p_{\text {int }}+\sum_{\substack{\gamma \in\{\mathrm{L}, \mathrm{G}, \mathrm{W}\} \\
\gamma \neq \beta}} \tau_{\beta \gamma} P_{\beta \gamma}=0
\end{gathered}
$$

where $\beta \in\{\mathrm{L}, \mathrm{G}\}$ denotes a phase, $t[\mathrm{~s}]$ is time, $s[\mathrm{~m}]$ is the pipe longitudinal distance, $\rho_{\beta}\left[\mathrm{kg} \mathrm{m} \mathrm{m}^{-3}\right]$ is the density of phase $\beta$, $u_{\beta}\left[\mathrm{m} \mathrm{s}^{-1}\right]$ is the average velocity of phase $\beta$ in longitudinal direction, $A_{\beta}\left[\mathrm{m}^{2}\right]$ is the area occupied by phase $\beta$ and $P_{\beta \gamma}[\mathrm{m}]$ is the length of the interface of phase $\beta$ with $\gamma \in\{\mathrm{L}, \mathrm{G}, \mathrm{W}\}$, where $\mathrm{W}$ denotes the pipe wall, $p_{\mathrm{av}, \beta}[\mathrm{Pa}]$ is the average pressure of phase $\beta, p_{\text {int }}[\mathrm{Pa}]$ is the pressure at the interface, $h_{\mathrm{int}}[\mathrm{m}]$ is the height of the interface with respect to the centre of the pipe, $r[\mathrm{~m}]$ is the radius of the pipe and $\tau_{\beta \gamma}\left[\mathrm{N} \mathrm{m}^{-2}\right]$ is the average interface stress between phase $\beta$ and phase or wall $\gamma$. For an illustration of some quantities, see Fig. 1 . The model is the result of applying cross-sectional averaging per phase of the three-dimensional conservation of mass and the Navier-Stokes equations. See [19] for the 


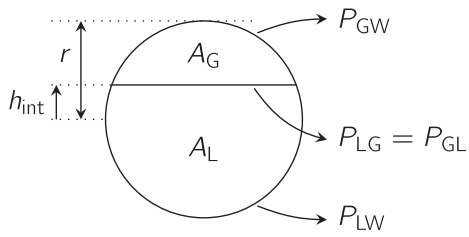

Fig. 1. Illustration of phase areas and perimeters as used in the two-fluid model.

derivation of this model. The model is similar to the models used by Liao et al. [6] and Fullmer et al. [8]. A difference worth noting is the hydrostatic pressure term $p_{\mathrm{av}, \beta}$ in the momentum Eq. (2), which is present due to the compressibility of the phases.

The areas of the liquid and gas phase cross sections are respectively given by

$A_{\mathrm{L}}=r^{2} \arccos \left(-\frac{h_{\mathrm{int}}}{r}\right)+h_{\mathrm{int}} \sqrt{r^{2}-h_{\text {int }}^{2}}$,

and

$A_{\mathrm{G}}=r^{2} \arccos \left(\frac{h_{\mathrm{int}}}{r}\right)-h_{\mathrm{int}} \sqrt{r^{2}-h_{\mathrm{int}}^{2}}$.

The perimeters of the liquid-gas, liquid-wall and gas-wall interfaces are respectively given by

$P_{\mathrm{LG}}=P_{\mathrm{GL}}=2 \sqrt{r^{2}-h_{\mathrm{int}}^{2}}$,

$P_{\mathrm{LW}}=2 r \arccos \left(-\frac{h_{\mathrm{int}}}{r}\right)$,

and

$P_{\mathrm{GW}}=2 r \arccos \left(\frac{h_{\mathrm{int}}}{r}\right)$.

The hydrostatic pressure integrated over the liquid and gas phase areas are respectively given by

$A_{\mathrm{L}} p_{\mathrm{av}, \mathrm{L}}=p_{\mathrm{int}} A_{\mathrm{L}}+\rho_{\mathrm{L}} g\left(h_{\mathrm{int}} A_{\mathrm{L}}+\frac{1}{12} P_{\mathrm{LG}}^{3}\right)$

and

$A_{G} p_{\mathrm{av}, \mathrm{G}}=p_{\mathrm{int}} A_{\mathrm{G}}+\rho_{\mathrm{G}} g\left(h_{\mathrm{int}} A_{\mathrm{G}}-\frac{1}{12} P_{\mathrm{LG}}^{3}\right)$,

where $g\left[\mathrm{~m} \mathrm{~s}^{-2}\right]$ is the gravitational acceleration.

The shear stress term $\tau_{\beta \gamma}, \beta \in\{\mathrm{G}, \mathrm{L}\}$ is physically modelled by the correlations of Taitel and Dukler [20]:

$\tau_{\beta \gamma}= \begin{cases}\frac{1}{2} f_{\beta} \rho_{\beta} u_{\beta}\left|u_{\beta}\right| & \text { if } \gamma=\mathrm{W} \\ \frac{1}{2} f_{\text {int }} \rho_{\mathrm{G}}\left(u_{\beta}-u_{\gamma}\right)\left|u_{\beta}-u_{\gamma}\right| & \text { if } \gamma \in\{\mathrm{G}, \mathrm{L}\},\end{cases}$

where $\mu_{\beta}$ [Pas] is the dynamic viscosity of phase $\beta$, the friction factor $f$ at the phase-wall interfaces and the gas-liquid interface are respectively given by

$f_{\beta}=0.046\left(\frac{\left|u_{\beta}\right| D_{\beta} \rho_{\beta}}{\mu_{\beta}}\right)^{0.2}, \quad \beta \in\{\mathrm{L}, \mathrm{G}\}$,

and

$f_{\text {int }}=\max \left\{f_{\mathrm{G}}, 0.014\right\}$,

and the hydraulic diameters $D_{\beta}$ are given by

$D_{\beta}= \begin{cases}\frac{4 A_{\mathrm{L}}}{P_{\mathrm{LW}}} & \text { if } \beta=\mathrm{L}, \\ \frac{4 A_{\mathrm{G}}}{P_{\mathrm{GW}}+P_{\mathrm{GL}}} & \text { if } \beta=\mathrm{G} .\end{cases}$
The two-fluid model is closed by defining the density $\rho_{\beta}$ and the viscosity $\mu_{\beta}$ for each phase $\beta$ and the pipe radius $r$. Those values are specific to a test case and are defined in Section 6 where the numerical results are discussed. The remaining unknowns are the interface pressure $p_{\text {int }}$, the interface height $h_{\text {int }}$ and the phase velocities $u_{\mathrm{L}}$ and $u_{\mathrm{G}}$.

\subsection{Homogeneous equilibrium model}

The second model considered is the homogeneous equilibrium model. That model is based on the assumption that the two phases are mixed and flow with a single mixture velocity, $u_{\mathrm{M}}\left[\mathrm{m} \mathrm{s}^{-1}\right]$. Let $A_{\mathrm{M}}\left[\mathrm{m}^{2}\right]$ denote the area of the pipe cross section,

$A_{\mathrm{M}}=\pi r^{2}$,

and $\alpha_{\beta}$ the holdup of phase $\beta$, with the constraint that the holdups sum to one,

$\alpha_{\mathrm{L}}+\alpha_{\mathrm{G}}=1$.

The mass balance equations will then read:

$\partial_{t}\left(A_{\beta} \rho_{\beta}\right)+\partial_{s}\left(A_{\beta} \rho_{\beta} u_{M}\right)=0$,

with the phase areas defined by

$A_{\beta}=A_{\mathrm{M}} \alpha_{\beta}$.

Compared to the two-fluid model only one, total momentum balance equation remains:

$\partial_{t}\left(A_{\mathrm{M}} \rho_{\mathrm{M}} u_{\mathrm{M}}\right)+\partial_{s}\left(A_{\mathrm{M}} \rho_{\mathrm{M}} u_{\mathrm{M}}^{2}+A_{\mathrm{M}} p_{\mathrm{M}}\right)=-\tau_{\mathrm{MW}} P_{\mathrm{M}}$,

where $\tau_{\mathrm{MW}}\left[\mathrm{N} \mathrm{m}^{-2}\right]$ is the wall friction of the mixture, $p_{\mathrm{M}}[\mathrm{Pa}]$ is the mixture pressure and $\rho_{\mathrm{M}}\left[\mathrm{kg} \mathrm{m}^{-3}\right]$ is the mixture density, an area-weighted average of the phase densities,

$\rho_{\mathrm{M}}=\alpha_{\mathrm{L}} \rho_{\mathrm{L}}+\alpha_{\mathrm{G}} \rho_{\mathrm{G}}$.

The total momentum Eq. (18) is conservative, whereas the momentum per phase Eq. (2) for the two-fluid model is not conservative.

For the wall friction we use Churchill's friction factor. The wall shear stress is given by

$\tau_{\mathrm{MW}}=\frac{1}{2} f_{\mathrm{MW}} \rho_{\mathrm{M}} u_{\mathrm{M}}\left|u_{\mathrm{M}}\right|$,

with friction factor $f_{\mathrm{MW}}$ given by

$f_{\mathrm{MW}}=2\left(\left(\frac{8}{\operatorname{Re}}\right)^{12}+\left(\Theta_{1}+\Theta_{2}\right)^{-1.5}\right)^{\frac{1}{12}}$,

parameters $\Theta_{1}$ and $\Theta_{2}$ given by

$\Theta_{1}=\left(-2.457 \ln \left(\left(\frac{7}{\operatorname{Re}}\right)^{0.9}+0.27 \frac{\epsilon_{\text {pipe }}}{2 r}\right)\right)^{16}$,

and

$\Theta_{2}=\left(\frac{37530}{\operatorname{Re}}\right)^{16}$,

where $\epsilon_{\text {pipe }}$ is the pipe roughness. The Reynolds number of the mixture is defined as

$\operatorname{Re}=\frac{2 r \rho_{\mathrm{M}} u_{\mathrm{M}}}{\mu_{\mathrm{M}}}$,

and the mixture dynamic viscosity is defined as the area-weighted average of the phase viscosities,

$\mu_{\mathrm{M}}=\alpha_{\mathrm{L}} \mu_{\mathrm{L}}+\alpha_{\mathrm{G}} \mu_{\mathrm{G}}$.

The Homogeneous equilibrium model is closed by defining the density $\rho_{\beta}$ and the viscosity $\mu_{\beta}$ for each phase $\beta$, the pipe radius $r$ and the pipe roughness $\epsilon_{\text {pipe. }}$. Those values are specific to a test case and are defined in Section 6 where the numerical results are discussed. The remaining unknowns are the mixture pressure $p_{\mathrm{M}}$, the liquid holdup $\alpha_{\mathrm{L}}$ and the mixture velocity $u_{\mathrm{M}}$. 


\section{Discontinuous Galerkin discretisation of the two-fluid model}

In this section we derive the space-time DG discretisation for both models given in Section 2, expressed in general form as

$\partial_{t} f_{\mathrm{t} j}(q)+\partial_{s} f_{\mathrm{s} j}(q)+\sum_{k} F_{\mathrm{s} j k}(q) \partial_{s} q_{k}-\partial_{s}\left(D_{j} \partial_{s} f_{\mathrm{t} j}(q)\right)+g_{j}(q)=0$,

where $s \in \mathcal{S} \subseteq \mathbb{R}$ refers to space and $t \in \mathcal{T}:=[0, T]$ to time, $q$ : $\mathcal{S} \times \mathcal{T} \rightarrow \mathbb{R}^{N}$ is the vector of unknowns as a function of space-time position, $f_{\mathrm{t}}: \mathbb{R}^{N} \rightarrow \mathbb{R}^{N}$ the mapping from unknowns to conserved quantities, $f_{\mathrm{s}}: \mathbb{R}^{N} \rightarrow \mathbb{R}^{N}$ the conservative part of the spatial flux, $F_{\mathrm{S}}: \mathbb{R}^{N} \rightarrow \mathbb{R}^{N \times N}$ the non-conservative part of the spatial flux and $g: \mathbb{R}^{N} \rightarrow \mathbb{R}^{N}$ the source term. For brevity the arguments $s$ and $t$ of $q$ are omitted here and in the following. The diffusion coefficients $D: \mathbb{R}^{N}$ are introduced for stability and will be discussed in Section 3.4.

\subsection{Weak formulation}

Let $\left(s_{a}, s_{b}\right) \times\left(t_{a}, t_{b}\right) \subseteq \mathcal{S} \times \mathcal{T}$ be a rectangular space-time element. Let $v: \mathcal{S} \times \mathcal{T} \rightarrow \mathbb{R}$ be a function on the space-time domain. Multiplying the general PDE (26) with test function $v$ and integrating over the element gives

$$
\begin{gathered}
\int_{s_{a}}^{s_{b}} \int_{t_{a}}^{t_{b}} v\left(\partial_{t} f_{\mathrm{t} j}(q)+\partial_{s} f_{s j}(q)+\sum_{k} F_{s j k}(q) \partial_{s} q_{k}\right) d t d s \\
+\int_{s_{a}}^{s_{b}} \int_{t_{a}}^{t_{b}} v\left(-\partial_{s}\left(D_{j} \partial_{s} f_{\mathrm{t} j}(q)\right)+g_{j}(q)\right) d t d s=0 .
\end{gathered}
$$

Integration by parts of the first, second and fourth term yields

$$
\begin{aligned}
& \int_{s_{a}}^{s_{b}} \int_{t_{a}}^{t_{b}}\left(-\partial_{t} v f_{\mathrm{t} j}(q)-\partial_{s} v f_{s j}(q)\right) d t d s \\
& +\int_{s_{a}}^{s_{b}} \int_{t_{a}}^{t_{b}} v \sum_{k} F_{s j k}(q) \partial_{s} q_{k} d t d s \\
& +\int_{s_{a}}^{s_{b}} \int_{t_{a}}^{t_{b}}\left(\partial_{s} v D_{j} \partial_{s} f_{\mathrm{t} j}(q)+v g_{j}(q)\right) d t d s \\
& +\left[\int_{s_{a}}^{s_{b}} v^{\text {in }} f_{\mathrm{t} j}\left(q^{\mathrm{in}}\right) d s\right]_{t=t_{a}}^{t_{b}}+\left[\int_{t_{a}}^{t_{b}} v^{\text {in }} f_{s j}\left(q^{\mathrm{in}}\right) d t\right]_{s=s_{a}}^{s_{b}} \\
& +\left[\int_{t_{a}}^{t_{b}} v^{\text {in }} D_{j} \partial_{s} f_{\mathrm{t} j}\left(q^{\mathrm{in}}\right) d t\right]_{s=s_{a}}^{s_{b}}=0 .
\end{aligned}
$$

The superscript 'in' denotes the trace of a function from within the element, formally

$q^{\text {in }}(s, t):=\lim _{\epsilon \rightarrow 0^{+}} q\left(s-\epsilon n_{\mathrm{s}}(s, t), t-\epsilon n_{\mathrm{t}}(s, t)\right)$,

with $n_{\mathrm{s}}, n_{\mathrm{t}}$ the unit outward normal of the element. The value at the opposite side of the element boundary is denoted with superscript 'out':

$q^{\text {out }}(s, t):=\lim _{\epsilon \rightarrow 0^{+}} q\left(s+\epsilon n_{\mathrm{s}}(s, t), t+\epsilon n_{\mathrm{t}}(s, t)\right)$.

\subsubsection{Temporal flux}

For the temporal flux at the time boundary we use plain upwinding. This amounts to replacing $q^{\text {in }}$ in the $t_{a}$-boundary integral of Eq. (28) with $q^{\text {out }}$. The total time flux boundary contribution becomes

$R_{\mathrm{tf}}:=\left[\int_{s_{a}}^{s_{b}} v^{\text {in }} f_{\mathrm{t} j}\left(q^{\mathrm{in}}\right) d s\right]_{t=t_{b}}-\left[\int_{s_{a}}^{s_{b}} v^{\text {in }} f_{\mathrm{t} j}\left(q^{\text {out }}\right) d s\right]_{t=t_{a}}$,

replacing the second term in Eq. (28).

\subsubsection{Spatial flux}

The treatment of the spatial flux at the spatial element boundaries is based on an approximate Riemann solver. Since the system of PDEs is non-conservative, at least for the first model described in Section 2, standard Riemann solvers cannot be applied. Vol'pert [21] studied non-conservative systems and interpreted the non-conservative product as a product of a function with a measure. Dal Maso et al. [22] generalised this interpretation of the non-conservative product, known as the DLM-measure. At a discontinuity the non-conservative product is defined as the integral of $F^{\text {total }}$ over a path connecting both ends of the discontinuity. Given a family of integration paths, this gives a rigorous definition of weak solutions to the non-conservative system. These weak solutions, however, depend on the chosen integration path. See for example Chalmers and Lorin [23] for a discussion on choosing appropriate integration paths. Several conservative numerical schemes and approximate Riemann solvers have been generalised to non-conservative systems based on the theory by Dal Maso et al. [22]: Lax-Friedrichs and Lax-Wendroff [24], Roe's approximate Riemann solver [25], HLL [26] and the Osher Riemann solver [27]. Parés [28] introduced the concept of path-conservative numerical schemes, as a generalisation of conservative schemes.

Due to the rather complex spatial flux of the two-fluid model defined in Section 2 we did not consider deriving an analytical expression of the eigenvalues and eigenvectors of $F^{\text {total }}$. Instead we rely on numerical computation. Since the Osher Riemann solver [27] requires the eigenstructure to be known along the integration paths connecting both ends of discontinuities, we deemed this method too expensive. The simpler Lax-Friedrichs method is in our experience not stable enough for the PDEs considered in this article. We settled for a linearised Riemann solver based on Roe's approach [25], which requires a single numerical evaluation of the eigenvalues and eigenvectors per spatial boundary point, but we replace Roe's matrix with $F_{s}^{\text {total }}\left(q^{\text {av }}\right)$, where $q^{\text {av }}$ is the average value of the inner and outer trace,

$q_{j}^{\text {av }}=\frac{1}{2}\left(q_{j}^{\text {in }}+q_{j}^{\text {out }}\right), \forall j$.

Let $F_{\mathrm{s}}^{\text {total }}: \mathbb{R}^{N} \rightarrow \mathbb{R}^{N \times N}$ be the total spatial flux matrix, combining the conservative flux Jacobian with the non-conservative flux matrix:

$F_{\mathrm{s} j l}^{\text {total }}(q)=\partial_{q_{l}} f_{\mathrm{s} j}(q)+F_{\mathrm{s} j l}(q)$,

and let $F_{\mathrm{t} j l}: \mathbb{R}^{N} \rightarrow \mathbb{R}^{N \times N}$ be the temporal flux Jacobian:

$F_{\mathrm{t} j l}(q)=\partial_{q_{l}} f_{\mathrm{t} j}(q)$

Let $\lambda_{k}$ and $X_{j k}$ be the $k$ th eigenvalue and eigenvector of the generalised eigenvalue problem:

$\sum_{l} F_{\mathrm{s} j l}^{\mathrm{total}}\left(q^{\mathrm{av}}\right) X_{l k}=\sum_{l} F_{\mathrm{t} j l}\left(q^{\mathrm{av}}\right) X_{j k} \lambda_{k} \forall k$

Solving the linearised Riemann problem and selecting the centre state yields

$q_{j}^{*}=q_{j}^{\text {in }}+\sum_{k, l \text { if } \lambda_{k} n_{\mathrm{s}}<0} X_{j k} X_{k l}^{-1}\left(q_{l}^{\text {out }}-q_{l}^{\text {in }}\right)$.

This definition of $q^{*}$ only applies for internal element boundaries. The domain boundary conditions are described in Section 3.3. Before continuing we need the following definition of integration paths:

Definition 1 (Integration paths, multidimensional version [22]). A Lipschitz continuous path $\phi:[0,1] \times \mathbb{R}^{N} \times \mathbb{R}^{N} \rightarrow \mathbb{R}^{N}$ is called an integration path if it satisfies the following properties:

- The path defined by states $q^{-}$and $q^{+}$begins and ends in those states respectively: 


$$
\phi_{j}\left(0 ; q^{-}, q^{+}\right)=q_{j}^{-} \text {and } \phi_{j}\left(1 ; q^{-}, q^{+}\right)=q_{j}^{+} \quad \forall j, \forall q^{-}, q^{+} \in \mathbb{R}^{N} \text {. }
$$

- If both states are equal, the path is constant:

$$
\phi_{j}(\tau ; q, q)=q_{j} \forall j, \forall q \in \mathbb{R}^{N}, \tau \in[0,1] .
$$

- For every bounded set $\mathcal{U}$ of $\mathbb{R}^{N}$, there exists $k \geq 1$ such that

$$
\begin{aligned}
& \left|\partial_{\tau} \phi\left(\tau ; q^{-}, q^{+}\right)-\partial_{\tau} \phi\left(\tau ; w^{-}, w^{+}\right)\right| \leq k\left|\left(q^{-}-w^{-}\right)-\left(q^{+}-w^{+}\right)\right| \\
& \forall q^{-}, q^{+}, w^{-}, w^{+} \in \mathcal{U}, \tau \text { a.e. } \in[0,1] .
\end{aligned}
$$

- Reversing the arguments reverses the path:

$$
\phi\left(\tau ; q^{-}, q^{+}\right)=\phi\left(1-\tau ; q^{+}, q^{-}\right) \forall q^{-}, q^{+} \in \mathbb{R}^{N}, \tau \in[0,1] .
$$

Proceeding with Roe's approximate Riemann solver the contribution of the spatial flux flowing inward is given by the following term

$\left[\int_{t_{a}}^{t_{b}} v^{\text {in }} \int_{0}^{1} \sum_{k} F_{s j k}^{\text {total }}(\phi) \partial_{s} \phi_{k} d \tau d t\right]_{s=s_{a}}^{s_{b}}$,

with $\phi=\phi\left(\tau ; q^{\text {in }}, q^{*}\right)$. By Definition 1 and Eq. (33) this can be simplified to

$$
\begin{aligned}
R_{\mathrm{sf}} & {\left[\int_{t_{a}}^{t_{b}} v^{\text {in }} f_{s j}\left(q^{\mathrm{in}}\right) d t\right]_{s=s_{a}}^{s_{b}} } \\
:= & {\left[\int_{t_{a}}^{t_{b}} v^{\text {in }}\left(f_{\mathrm{s} j}\left(q^{*}\right)-f_{\mathrm{sj}}\left(q^{\mathrm{in}}\right)\right) d t\right]_{s=s_{a}}^{s_{b}} } \\
& +\left[\int_{t_{a}}^{t_{b}} v^{\text {in }} \int_{0}^{1} \sum_{k} F_{\mathrm{s} j k}(\phi) \partial_{s} \phi_{k} d \tau d t\right]_{s=s_{a}}^{s_{b}} .
\end{aligned}
$$

This term is to be added to the left hand side of Eq. (28).

We assume a linear path connecting the states $q^{\text {in }}$ and $q^{*}$ :

$\phi_{j}\left(\tau ; q^{\text {in }}, q^{*}\right):=q_{j}^{\text {in }}(1-\tau)+q_{j}^{*} \tau$.

The choice of the integration path affects the solution to the discrete system. Rhebergen et al. [26] have investigated the effect of the path on the numerical solution, in particular the shock speed, for a similar two-fluid model and have concluded that different paths lead only to minimal changes in the solution. Furthermore, they note that for a linear path a low-order Gauss integration scheme is sufficient and yields the most computationally efficient scheme.

\subsubsection{Diffusion}

Following the DGFEM formulation of Baumann and Oden [29] for a convection-diffusion model the last term of Eq. (28) is replaced by

$$
\begin{aligned}
R_{\mathrm{diff}}:= & {\left[\int_{t_{a}}^{t_{b}}-\frac{1}{2} D_{j} \partial_{s}\left(v^{\text {out }}+v^{\text {in }}\right)\left(f_{\mathrm{t} j}\left(q^{\text {out }}\right)-f_{\mathrm{t} j}\left(q^{\text {in }}\right)\right) d t\right]_{s=s_{a}}^{s_{b}} } \\
& +\left[\int_{t_{a}}^{t_{b}} \frac{1}{2} D_{j} \partial_{s}\left(f_{\mathrm{t} j}\left(q^{\text {out }}\right)+f_{\mathrm{t} j}\left(q^{\text {in }}\right)\right)\left(v^{\text {out }}-v^{\text {in }}\right) d t\right]_{s=s_{a}}^{s_{b}} .
\end{aligned}
$$

Bassi and Rebay [30] have compared the stabilisation of the diffusion term from Eq. (44) with a more elaborate local DG type treatment [31] and they concluded that the latter is superior with respect to the accuracy on coarse meshes. However, due to the additional computational complexity we have chosen for the simpler option. For an overview of stabilisation methods for diffusion terms we refer the reader to Arnold et al. [32].

\subsubsection{Result}

Combining all additions and replacements defined above, the resulting weak formulation is given by

$$
\begin{aligned}
& \int_{s_{a}}^{s_{b}} \int_{t_{a}}^{t_{b}}\left(-\partial_{t} v f_{\mathrm{t} j}(q)-\partial_{s} v f_{\mathrm{s} j}(q)\right) d t d s \\
& \quad+\int_{s_{a}}^{s_{b}} \int_{t_{a}}^{t_{b}} v \sum_{k} F_{\mathrm{s} j k}(q) \partial_{s} q_{k} d t d s \\
& \quad+\int_{s_{a}}^{s_{b}} \int_{t_{a}}^{t_{b}}\left(-\partial_{s} v D_{j} \partial_{s} f_{\mathrm{t} j}(q)+v g_{j}(q)\right) d t d s+R_{\mathrm{tf}}+R_{\mathrm{sf}}+R_{\mathrm{diff}}=0 .
\end{aligned}
$$

\subsection{Mesh and basis}

We use a structured partition $\mathcal{E}$ of the space-time domain $\mathcal{S} \times \mathcal{T}$ as (coarse) mesh. For each element $E \in \mathcal{E}$ we define a local basis as a tensor product of one-dimensional Legendre basis functions with maximum order $p$ for space and time, with support limited to element $E$. The basis $\mathcal{Q}$ is defined as union of all element bases.

Given a space-time mesh $\mathcal{E}$ and basis, solving the complete discrete system at once is in general too expensive and also unnecessary. We create a possibly finite sequence $\left\{\mathcal{E}_{0}, \mathcal{E}_{1}, \mathcal{E}_{2}, \ldots\right\}$ of subsets of $\mathcal{E}$, such that the sequence is a partition of $\mathcal{E}$ and all elements of $\mathcal{E}_{k}$ are a subset of time-interval $\mathcal{S} \times\left[t_{k}, t_{k_{1}}\right]$. Let $\mathcal{Q}_{k}$ be the subset of basis functions with support on time slab $k$. Given a sequence of time slabs, we can solve each time slab one after another due to upwinding in time (Section 3.1.1).

\subsection{Boundary conditions}

For the boundary conditions, if present, we use the same machinery as introduced in Section 3.1.2 for the internal element boundaries. In absence of an outer value $q^{\text {out }}$, the linearisation state $q^{\mathrm{av}}$, introduced in Section 3.1, is chosen equal to the inner value $q^{\text {in }}$.

Assume that there are $N_{\mathrm{L}}$ problem specific (external) boundary conditions at the left boundary and $N_{\mathrm{R}}$ at the right boundary, respectively given by the following roots

$f_{j \mathrm{~L}}\left(q^{*}\right)=0, \quad j \in\left\{0,1, \ldots, N_{\mathrm{L}}-1\right\}$,

and

$f_{j \mathrm{R}}\left(q^{*}\right)=0, \quad j \in\left\{0,1, \ldots, N_{\mathrm{R}}-1\right\}$.

These boundary conditions are supplemented with the following (internal) outflow boundary conditions:

$\sum_{j} X_{k j}^{-1}\left(q_{j}^{*}-q_{j}^{\text {in }}\right)=0$ for all $k$ satisfying $\lambda_{k} n_{\mathrm{s}}>0$,

where eigenvalues $\lambda_{k}$ and eigenvectors $X_{j k}$ are defined by Eq. (35) and $n_{\mathrm{s}}$ is the spatial component of the unit outward normal. Note that the number of boundary conditions being the sum of all internal and external conditions, should equal the number of equations $n$.

\subsection{Artificial viscosity}

To incorporate artificial viscosity, we use the technique described by Persson and Peraire [17]. For each time-slab we initially solve the system without artificial viscosity. Then we add a sufficient amount of viscosity, via parameter $D$ of Eq. (26), such that the smoothness is above a threshold for all elements. The algorithm is illustrated in Fig. 2 with $K$ set to zero. The smoothness indicator is given by [17]

$\int_{s_{a}}^{s_{b}} \int_{t_{a}}^{t_{b}} \frac{\left|q_{j}-\hat{q}_{j}\right|^{2}}{\left|q_{j}\right|^{2}} d t d s$ 


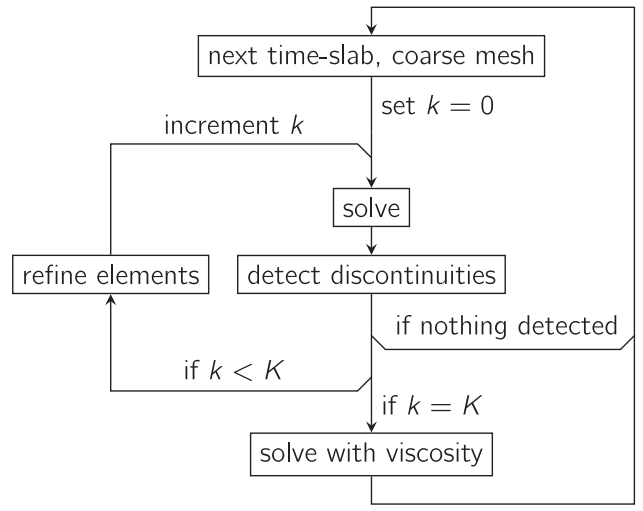

Fig. 2. DGFEM solver algorithm with a maximum of $K$ adaptive mesh refinements.

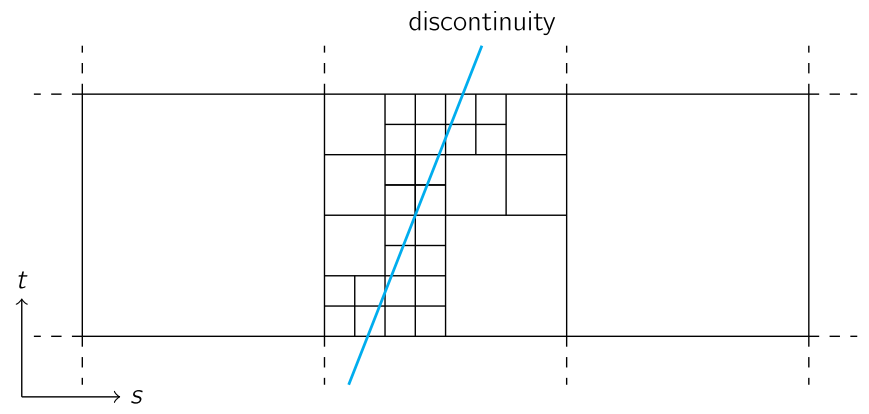

Fig. 3. Example of multilevel $h$-refinement near a discontinuity.

where $\hat{q}$ is equal to the solution $q$ projected onto a solution space with degree $p-1$, one degree lower than the solution space for $q$, hence the difference $q_{j}-\hat{q}_{j}$ represents the high-frequency part of the solution $q_{j}$ only.

\subsection{Local refinement}

Discontinuities reduce the (uniform) scheme to first order in mesh width. When using a high-order basis, uniform refinement is less effective when discontinuities are present. To increase the efficiency (in terms of the number of elements in time slab $k, \mathcal{E}_{k}$, or the number of basis functions in time slab $k, \# \mathcal{Q}_{k}$ ) we apply local mesh refinement in the neighbourhood of discontinuities several times, yielding a mesh where the element density increases gradually towards the discontinuity. A fictive example is given in Fig. 3.

The refinement scheme works as follows. For each time-slab we compute a solution on a coarse mesh without artificial viscosity. Based on the smoothness indicator we refine elements where the smoothness is below a threshold and recompute a solution on the refined mesh. The refinement step is repeated a predefined number of times $K$ or until all elements are smooth enough, whichever is reached first. Finally, we add viscosity to elements with a smoothness below a threshold and recompute a solution one more time. The complete algorithm with refinement and artificial viscosity is illustrated in Fig. 2.

\subsection{Implementation details}

The weak formulation is linearised by Newton's method. The jacobian is computed using (automated) symbolic differentiation and the linear system, part of Newton's method, is solved using UMFPACK [33]. The algorithm is implemented in Python and uses the finite element package Nutils. The implementation is available online [34].

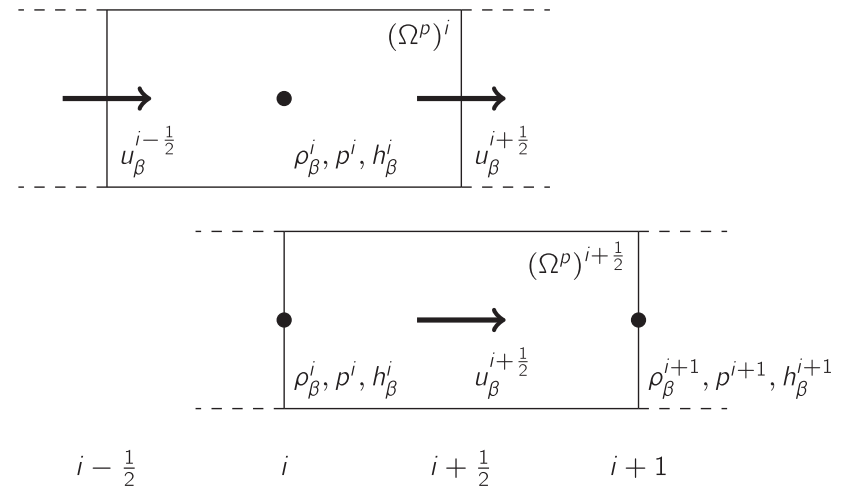

Fig. 4. Staggered grid layout of the FV scheme.

\section{Finite volume discretisation}

\subsection{Spatial discretization}

We discretise the two-fluid model, i.e. Eqs. (1) and (2), by using a finite volume method on a staggered grid. As indicated in Fig. 4, the staggered grid consists of both $p$-volumes, $\Omega^{p}$, and $u$ volumes, $\Omega^{u}$. Each volume consists of a liquid and a gas phase: $\Omega=\Omega_{\mathrm{L}} \cup \Omega_{\mathrm{G}}$, for both $u$ - and $p$-volumes. We start with conservation of mass for a phase $\beta$, ( $\beta$ is liquid or gas). Integration of Eq. (1) in $s$-direction gives:

$\partial_{t}\left(\left(\Omega_{\beta}^{p}\right)^{i} \rho_{\beta}^{i}\right)+\left(A_{\beta} \rho_{\beta}\right)^{i+1 / 2} u_{\beta}^{i+1 / 2}-\left(A_{\beta} \rho_{\beta}\right)^{i-1 / 2} u^{i-1 / 2}=0$,

with the finite volume size approximated by

$\left(\Omega_{\beta}^{p}\right)^{i}=A_{\beta}^{i} \Delta s_{p}^{i}$.

The finite volume size can be used to rewrite the semi-discrete equation for conservation of mass into:

$\partial_{t}\left(A_{\beta}^{i} \rho_{\beta}^{i}\right)+\frac{\left(A_{\beta} \rho_{\beta}\right)^{i+1 / 2} u_{\beta}^{i+1 / 2}-\left(A_{\beta} \rho_{\beta}\right)^{i-1 / 2} u_{\beta}^{i-1 / 2}}{\Delta s_{p}^{i}}=0$.

The term $\left(A_{\beta} \rho_{\beta}\right)^{i+1 / 2}$ requires interpolation from neighbouring values, which is described below. For conservation of momentum we proceed in a similar way. Integration of (2) in $s$-direction gives:

$$
\begin{aligned}
\partial_{t}\left(\left(\Omega_{\beta}^{u}\right)^{i+1 / 2} \rho_{\beta}^{i+1 / 2} u_{\beta}^{i+1 / 2}\right)+\left(A_{\beta} \rho_{\beta}\right)^{i+1}\left(u_{\beta}^{i+1}\right)^{2}-\left(A_{\beta} \rho_{\beta}\right)^{i}\left(u_{\beta}^{i}\right)^{2} \\
=-A_{\beta}^{i+1 / 2}\left(p^{i+1}-p^{i}\right)-\left(\rho_{\beta}^{i+1} \mathrm{LG}_{\beta}^{i+1}-\rho_{\beta}^{i} \mathrm{LG}_{\beta}^{i}\right) \\
\quad-\sum_{\substack{\gamma \in\{\mathrm{L}, \mathrm{G}, \mathrm{W}\} \\
\gamma \neq \beta}} \tau_{\beta \gamma}^{i+1 / 2} P_{\beta \gamma}^{i+1 / 2} \Delta s_{u}^{i+1 / 2},
\end{aligned}
$$

where

$\left(\Omega_{\beta}^{u}\right)^{i+1 / 2}=A_{\beta}^{i+1 / 2} \Delta s_{u}^{i+1 / 2}$,

and the level gradient terms are given by

$\mathrm{LG}_{\mathrm{G}}=h A_{\mathrm{G}}+\frac{1}{12} w^{3}, \quad \mathrm{LG}_{\mathrm{L}}=h A_{\mathrm{L}}-\frac{1}{12} w^{3}$.

The discretisation of the homogeneous equilibrium model, Eqs. (16) and (18), makes use of the same staggered grid layout. The semi-discrete equations for conservation of mass and momentum for this model will then read:

$\partial_{t}\left(A_{\beta}^{i} \rho_{\mathrm{M}}^{i}\right)+\frac{\left(A_{\beta} \rho_{\mathrm{M}}\right)^{i+1 / 2} u_{\mathrm{M}}^{i+1 / 2}-\left(A_{\beta} \rho_{\mathrm{M}}\right)^{i-1 / 2} u_{\mathrm{M}}^{i-1 / 2}}{\Delta s_{p}^{i}}=0$, 
and

$$
\begin{gathered}
\partial_{t}\left(A_{\mathrm{M}}^{i+1 / 2} \rho_{\mathrm{M}}^{i+1 / 2} u_{\mathrm{M}}^{i+1 / 2}\right)+\frac{\left(\left(A_{\mathrm{M}} \rho_{\mathrm{M}}\right)^{i+1}\left(u_{\mathrm{M}}^{i+1}\right)^{2}+\left(A_{\mathrm{M}} p\right)^{i+1}\right)}{\Delta s_{u}^{i+1 / 2}} \\
-\frac{\left(\left(A_{\mathrm{M}} \rho_{\mathrm{M}}\right)^{i}\left(u_{\mathrm{M}}^{i}\right)^{2}+\left(A_{\mathrm{M}} p\right)^{i}\right)}{\Delta s_{u}^{i+1 / 2}}=-\tau_{\mathrm{MW}}^{i+1 / 2} P_{\mathrm{M}}^{i+1 / 2} .
\end{gathered}
$$

Several terms in Eqs. (52), (53), (56) and (57) require approximation. All terms that are not part of the convective terms are interpolated using a central scheme, e.g. $A_{\beta}^{i+1 / 2}=\frac{1}{2}\left(A_{\beta}^{i}+A_{\beta}^{i+1}\right)$. The convective terms, on the other hand, require more care in order to prevent numerical oscillations. They are computed in an upwind fashion using a high resolution scheme as follows. Let $\phi$ denote a generic quantity on a cell face (either $u^{2}$ or $\rho A$ ) and let $\theta$ be a smoothness indicator, given by

$\theta^{i+1 / 2}=\frac{\phi_{c}-\phi_{u}}{\phi_{d}-\phi_{c}}$,

where

$\left[\phi_{\mathrm{u}}, \phi_{\mathrm{c}}, \phi_{\mathrm{d}}\right]= \begin{cases}{\left[\phi^{i-1}, \phi^{i}, \phi^{i+1}\right]} & \text { if } u^{i+1 / 2} \geq 0, \\ {\left[\phi^{i+2}, \phi^{i+1}, \phi^{i}\right]} & \text { if } u^{i+1 / 2}<0 .\end{cases}$

and $\phi_{d}, \phi_{u}$ and $\phi_{c}$ denote the downstream, upstream and central quantities of the face under consideration. The smoothness indicator is used to compute a slope-limiter $l(\theta)$, from which the face quantity follows as:

$\phi^{i+1 / 2}=\phi_{c}+\frac{1}{2} l^{i+1 / 2}\left(\phi_{d}-\phi_{c}\right)$.

In the current study the van Albada limiter,

$l(\theta)=\frac{\theta^{2}+\theta}{\theta^{2}+1}$,

has been used, mainly because of its continuous differentiability, which is a favourable property when the fully discrete equations are solved with a Newton solver.

\subsection{Boundary conditions}

Boundary conditions are set based on the characteristics of the system at the boundary [35]. To determine the characteristic equations, the system is written in quasi-linear form:

$\partial_{t} f_{\mathrm{t} j}(q)+\sum_{l} F_{s j l}^{\text {total }}(q) \partial_{s} q_{l}+g_{j}(q)=0$.

Defining $\lambda_{k}$ and $X_{j k}$ as the $k$-th eigenvalue and eigenvector of $F_{\text {sjl }}^{\text {total }}(q)$, see Eq. (35), we can write Eq. (62) as:

$\partial_{t} f_{\mathrm{tj}}(q)+\sum_{k} X_{j k} \ell_{k}+g_{j}(q)=0$,

where

$\ell_{k}=\lambda_{k} \sum_{l} X_{k l}^{-1} \partial_{s} q_{l}$.

Eq. (63) can now be used for time integration of the boundary points where boundary conditions are set through $\ell_{k}$ by making use of the sign of $\lambda_{k}$ at the boundary. At the left boundary outgoing waves are associated with negative eigenvalues while at the right boundary outgoing waves are associated with positive eigenvalues. In the case of outgoing waves, Eq. (64) can be used to calculate $\ell_{k}$ by approximating $\partial_{s} q_{l}$ with finite differences calculated from the interior of the domain. On the other hand, incoming waves are associated with positive eigenvalues at the left boundary and negative eigenvalues at the right boundary. In the case of incoming waves, $\ell_{k}$ can not be calculated from Eq. (64), rather it is set through the imposed boundary conditions at the left and right boundary. As an example we consider the homogeneous equilibrium model for which we can expect two positive eigenvalues and one negative eigenvalue assuming subsonic flow. This will lead to two incoming waves at the left boundary (inlet), which are determined from the time dependent boundary condition for the mass flow of the gas and the liquid by using Eq. (63) to solve for $\ell_{k}$. At the right boundary (outlet) we have one incoming wave, which is set by fixing the outlet pressure.

\subsection{Temporal discretization}

The semi-discrete equations of the two-fluid model (52) and (53) can be written in the form

$\partial_{t} f_{\mathrm{t} j}(q)=G_{j}(q)$.

The semi-discrete equations are solved with the BDF2 scheme (Backward Differentiation Formula):

$\frac{1}{\Delta t}\left(f_{\mathrm{t} j}\left(q^{n+1}\right)-\frac{4}{3} f_{\mathrm{t} j}\left(q^{n}\right)+\frac{1}{3} f_{\mathrm{t} j}\left(q^{n-1}\right)\right)=\frac{2}{3} G_{j}\left(q^{n+1}\right)$.

We have chosen the BDF2 scheme for the stability properties. The scheme is strongly A-stable (L-stable) which enables us to use large time steps at the cost of damping of fast transients. Eq. (66) forms a non-linear system of equations that is solved using a Newton approach:

$$
\begin{aligned}
& \sum_{k}\left[\frac{1}{\Delta t} \partial_{q_{j}} f_{\mathrm{t} k}\left(q^{m}\right)-\frac{2}{3} \partial_{q_{j}} G_{k}\left(q^{m}\right)\right] \Delta q_{k} \\
& =-\frac{1}{\Delta t}\left(f_{\mathrm{t} j}\left(q^{m}\right)-\frac{4}{3} f_{\mathrm{t} j}\left(q^{n}\right)+\frac{1}{3} f_{\mathrm{t} j}\left(q^{n-1}\right)\right)+\frac{2}{3} G_{j}\left(q^{m}\right) .
\end{aligned}
$$

To solve the non-linear system, we solve for the increments in the primitive variables $\Delta q$, but the final system that is solved is (66), and as a consequence mass and momentum will be conserved. The Jacobians $\partial_{q_{j}} f_{\mathrm{t} k}(q)$ and $\partial_{q_{j}} G_{k}(q)$ are computed automatically by using finite differences. The constraint in the form $A_{\mathrm{G}}=A-A_{\mathrm{L}}$ is used to close the system of equations. The time integration of the homogeneous equilibrium model (56) and (57) is done in the same way.

\section{Stability and well-posedness}

We introduce notions of stability and well-posedness in the general setting of the following quasilinear system of PDEs on infinite spatial domains,

$\sum_{l} F_{\mathrm{t} j l}(q) \partial_{t} q_{l}+\sum_{l} F_{s j l}(q) \partial_{s} q_{l}+g_{j}(q)=0$

where $q: \mathbb{R} \times[0, T] \rightarrow \mathbb{R}^{N}$ is a vector of quantities, $F_{\mathrm{t}}, F_{\mathrm{s}}: \mathbb{R}^{N} \rightarrow$ $\mathbb{R}^{N \times N}$ are matrices and $g: \mathbb{R}^{N} \rightarrow \mathbb{R}^{N}$ a vector. For readability the arguments $(s, t)$ of $q$ are omitted. Note that both models introduced in Section 2 can be written in this form. We assume that matrix $F_{\mathrm{t}}$ is invertible. However, at the location where one phase is vanishing the two-fluid model given above yields a singular matrix $F_{\mathrm{t}}$. This situation, which occurs when a full liquid slug body is formed, is not considered in this article. Instead we restrict the simulations to the formation and propagation of liquid hold-up waves, which never reach the top of the pipeline.

Assume $q$ is a solution to PDE (68) and constant in space and time. Adding a small perturbation $\epsilon: \mathbb{R} \times[0, T] \rightarrow \mathbb{R}^{N}$ to $q$ and linearising the PDE in $\epsilon$ around $q$ yields

$\sum_{l} F_{\mathrm{t} j l}(q) \partial_{t} \epsilon_{l}+\sum_{l} F_{s j l}(q) \partial_{s} \epsilon_{l}+\sum_{l} \partial_{q_{l}} g_{j}(q) \epsilon_{l}=0$.

Again, for readability we omit arguments $(s, t)$ of $\epsilon$. Solutions to this linear system of PDEs are of the form

$\epsilon_{j}(s, t)=r_{j} e^{i(k s-\omega t)}$, 
where $r \in \mathbb{C}^{N}$ is a vector, $k \in \mathbb{R}$ a wave-number and $\omega \in \mathbb{C}$. Substituting the solution (70) into PDE (69), moving the first term to the right hand side and dividing by $i$ yields the following generalised eigenvalue problem with eigenvalue $\omega$ and eigenvector $r$,

$\sum_{l}\left(k F_{\mathrm{s} j l}(q)-i \partial_{q_{l}} g_{j}(q)\right) r_{l}=\omega \sum_{l} F_{\mathrm{t} j l}(q) r_{l}$.

For a fixed wave-number $k$ all eigenvalues $\omega$ and eigenvectors $r$ satisfying this equation define non-trivial solutions to the linearised PDE (69).

Based on the solution (70) we define:

Definition 2 (growth, dissipation). Growth (in time) is the real part of $-i \omega$, or equivalently the imaginary part of $\omega$. Dissipation is the imaginary part of $-\omega$.

Definition 3 (dispersion). Dispersion is the imaginary part of $-i \omega$, or equivalently the real part of $-\omega$.

The system of PDEs (68) is called stable at $q$ if there is no (strictly positive) growth, i.e. for all wave-numbers $k$ all eigenvalues $\omega$ of characteristic Eq. (69) satisfy $\operatorname{Im} \omega \leq 0$. The system is called well-posed if the growth is bounded for all wave-numbers $k$. An equivalent condition is that all eigenvalues $\lambda$ of the following generalised eigenvalue problem are real,

$\sum_{l} F_{\mathrm{s} j l}(q) r_{l}=\lambda \sum_{l} F_{\mathrm{t} j l}(q) r_{l}$

For models without source terms the notions of well-posedness and stability coincide, i.e. the system is either stable and wellposed or unstable and ill-posed. To see this, note that the characteristic equations, Eqs. (71) and (72), are equivalent, with

$\lambda=\frac{\omega}{k}$,

If $\operatorname{Im} \lambda>0$, then $\operatorname{Im} \omega$ goes to positive infinity for the wave-number $k$ going to infinity, which implies unbounded growth, hence the system is ill-posed.

Both the two-fluid model and the homogeneous equilibrium model have no source terms in case the phases are inviscid.

The homogenous equilibrium model is unconditionally stable. The two-fluid model, however, is not unconditionally stable. When the slip velocity, the velocity difference between the two phases, becomes too large the model becomes ill-posed [6]. This is a known problem of the two-fluid model. We refer the reader to Prosperetti [36] for an analysis.

\subsection{Analysis of the DGFEM scheme}

In this section we analyse the effect of the DGFEM scheme on eigenvalue $\omega$. We were unable to find the convergence rates for the eigenvalues $\hat{\omega}$ of the system obtained by applying the spatial part of the DGFEM scheme. However, Ainsworth [37] was able to prove convergence of the wave-number in otherwise the same setting. The imaginary part of the wave number converges with order $2 p+2$ in mesh width and the real part with order $2 p+3$. Based on this result and because the two problems are very related we expect similar convergence behaviour of the eigenvalues.

For the analysis of the temporal part we continue with the evolution of a single characteristic wave with eigenvalue $\hat{\omega}$ of the spatial part,

$\partial_{t} w(t)=-i \hat{\omega} w(t)$,

which admits the following solution:

$w(t)=w(0) e^{-i \hat{\omega} t}, \quad t>0$.
Multiplying this equation with a test function, integrating over temporal element $b$ with length $\Delta t$ and multiplying with the inverse of the mass matrix yields

$\sum_{m} T_{l m, 0} \tilde{w}_{m b}+\sum_{m} T_{l m,-1} \tilde{w}_{m, b-1}=-i \hat{\omega} \tilde{w}_{l b}$,

where $\tilde{w}_{b} \in \mathbb{C}^{p+1}$ is the vector of coefficients representing $w$ in element $b$ and matrices $T_{l m, 0}, T_{l m,-1} \in \mathbb{R}^{(p+1) \times(p+1)}$ represent the time derivative, acting respectively on the solution of element $b$ and the previous element. Moving the first term to the right hand side and multiplying the equation with the inverse of

$M_{l m}:=T_{l m 0}+i \hat{\omega} \delta_{l m}$

gives

$\tilde{w}_{j b}=\sum_{l m} M_{j l}^{-1} T_{l m,-1} \tilde{w}_{m, b-1}$.

Since the solution at element $b$ depends only on the solution at the downwind end of the previous element we can restrict the discrete evolution equation (78) to downwind ends. Let $R \in \mathbb{R}^{p+1}$ be the restriction of a coefficient vector $\tilde{w}$ to downwind ends and $E \in \mathbb{R}^{p+1}$ any expansion of value to a coefficient vector such that the restriction of the expansion is one,

$\sum_{l} R_{l} E_{l}=1$.

Multiplying Eq. (78) with $R$ and replacing $\tilde{w}_{b}$ with the expansion of a scalar $\check{w} \in \mathbb{C}$ gives the scalar equation

$\check{w}_{b}=G(\hat{\omega}, \Delta t) \check{w}_{b-1}=-\sum_{l m} R_{j} M_{j l}^{-1} T_{l m} E_{m} \check{w}_{b-1}$.

Lesaint and Raviart [38] have analysed this DGFEM scheme and proved the following convergence theorem:

Theorem 1 (Convergence of downwind end values [38]). The DGFEM scheme (76) converges globally with order $2 p+1$ in time step size $\Delta t$, i.e. the error after one step is

$\left|G(\hat{\omega}, \Delta t)-e^{-i \hat{\omega} \Delta t}\right|=O\left(\Delta t^{2 p+2}\right)$.

This gives the following convergence result for the eigenvalues $\check{\omega}$ of the discrete system:

Corollary 1 (Convergence of eigenvalues of discrete system).

$|\check{\omega}-\hat{\omega}|=O\left(\Delta t^{2 p+1}\right)$

Furthermore, Lesaint and Raviart [38] showed that the DGFEM scheme is strongly A-stable, or L-stable.

\section{Numerical results}

We analyse the proposed DGFEM scheme using two test cases. In Section 6.1 we present a Kelvin-Helmholtz test case and verify the theoretical stability results presented in Section 5.1. This test case refers to the wave formation at the interface of the flow of air and water in a horizontal pipe at atmospheric pressure. In Section 6.2 we analyse the performance of the DGFEM scheme with and without adaptive refinement.

\subsection{Stability analysis using Kelvin-Helmholtz test case}

In this section we verify the theoretical results of Section 5 for the two-fluid model discretised with the uniform DG and FV schemes by comparing the theoretical and observed growth rate and dispersion of small sinusoidal waves on infinite domains.

As initial condition we use a constant reference state $q_{\text {ref }} \in \mathbb{R}^{N}$ that satisfies the system of PDEs with a sinusoidal perturbation with magnitude $c$,

$q_{\text {initial }, j}(s)=q_{\text {ref }, j}+c \operatorname{Re}\left(r_{j} e^{i k s}\right)$, 
where $r \in \mathbb{R}^{N}$ is a unit eigenvector of the system linearised around $q_{\text {ref }}$, see Eq. (69), and $k$ is a wave-number. As a reference solution we use the exact solution to the linearised model, given by

$q_{\text {lin }, j}(s, t)=q_{\text {ref, } j}+c \operatorname{Re}\left(\mathrm{r}_{\mathrm{j}} \mathrm{e}^{\mathrm{i}(\mathrm{ks}-\omega \mathrm{t})}\right)$,

where $\omega \in \mathbb{C}$ is the eigenvalue corresponding to the eigenvector $r$. This is close to the real solution when the amplitude $c$ is very small.

We start the analysis of the uniform DG and FV schemes with the inviscid two-fluid model. We use the following reference state,

$q_{\mathrm{ref}}=\left[\begin{array}{c}p_{\text {int }} \\ h_{\text {int }} \\ u_{\mathrm{L}} \\ u_{\mathrm{G}}\end{array}\right]=\left[\begin{array}{c}10^{5} \mathrm{~Pa} \\ 0 \mathrm{~m} \\ 1 \mathrm{~m} \mathrm{~s}^{-1} \\ 15 \mathrm{~m} \mathrm{~s}^{-1}\end{array}\right]$,

and the following model parameters: pipe radius $r=0.039 \mathrm{~m}$, gas density $\rho_{\mathrm{G}}=1.1614 \cdot 10^{-5} \mathrm{p}$ in $\mathrm{kg} \mathrm{m} \mathrm{m}^{-3}$, liquid density $\rho_{\mathrm{L}}=$ $1000 \mathrm{~kg} \mathrm{~m}^{-3}$, gravitational acceleration $g=9.8 \mathrm{~m} \mathrm{~s}^{-2}$ and viscosity is set to zero. Note that any choice for the reference state $q_{\text {ref }}$ would be an equilibrium solution of the two-fluid model, because the source terms, friction and longitudinal gravity forces, are absent. Since there is no viscosity, the model is either stable and well-posed or unstable and ill-posed. In this case the chosen reference state is in the stable region, but close to the ill-posed region.

At the reference state the two-fluid model has two large - in magnitude - eigenvalues, associated with pressure waves, and two significantly smaller eigenvalues, associated with mass transport. For oil and gas applications - our main interest - the latter is more relevant. Of the remaining two small eigenvalues we choose one, but note that the following results hold equally true for the other.

We choose $k=2 \pi$ and let $\omega$ and $r$ be the third (algebraically) eigenvalue and eigenvector of the system linearised around $q_{\text {ref }}$ :

$\omega=8.070 \ldots \cdot 10^{0}$,

and

$r=\left[\begin{array}{c}-9.980 \ldots \cdot 10^{-1} \\ 1.394 \ldots \cdot 10^{-4} \\ 1.294 \ldots \cdot 10^{-3} \\ 6.255 \ldots \cdot 10^{-2}\end{array}\right]$.

Trailing dots indicate that the displayed value is rounded. The amplitude of the perturbation $c$ is chosen such that the amplitude of the liquid holdup perturbation is $10^{-10}$ for DG and $10^{-6}$ for FV. We use a smaller perturbation for DG and quad precision arithmetic because for the high-order DG scheme we would not able to observe the expected rate of convergence otherwise. In absence of friction the imaginary part of $\omega$ is zero, hence the amplitude of the perturbation should remain constant.

Let the relative error be the $L_{2}$-norm of the difference between the discrete solution and the reference solution divided by the $L_{2}$ norm of the reference solution. For FV we use the $l_{2}$-norm instead of the $L_{2}$-norm. Fig. 5 shows the relative error of the liquid holdup at $t=1$, obtained using the second order FV scheme and the DG scheme with bases of order $p$, i.e. $(p+1)^{2}$ basis functions per element. The horizontal axis shows the square root of the average space-time density of the number of degrees of freedom, abbreviated as sqrtdofs, required to represent the discrete solution on the space-time domain $[0,1] \times[0,1]$. For the uniform DG scheme the number of degrees of freedom is inversely proportional to the area of an element and the square root (sqrtdofs) inversely proportional to the width of an element, assuming a fixed aspect ratio of the elements. For both schemes we set $\Delta t=\Delta s$. Reducing the element width $\Delta s$ with a factor of two increases the sqrtdofs by a factor two. A second order scheme theoretically reaches second order convergence with respect to $\Delta s$, hence order minus

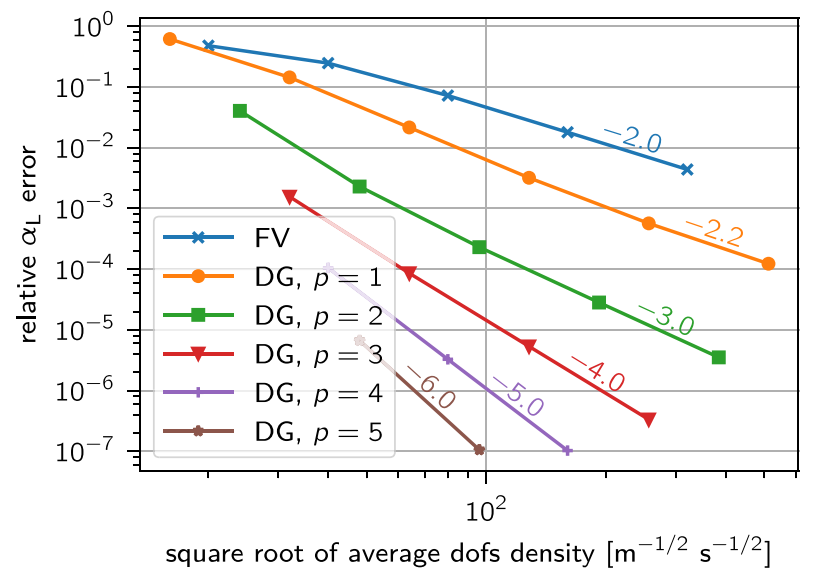

Fig. 5. Convergence of the relative error of the liquid holdup for the linear, inviscid Kelvin-Helmholtz test-case.

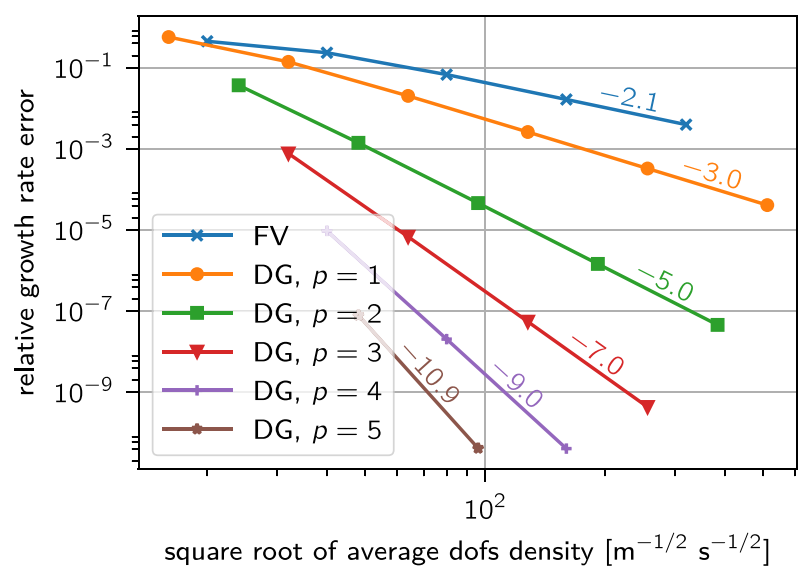

Fig. 6. Convergence of the relative error of the growth rate at $t=1 \mathrm{~s}$ for the linear, inviscid Kelvin-Helmholtz test-case.

two in terms of sqrtdofs. All schemes converge with the theoretical rate. We deliberately chose sqrtdofs as measure over the mesh width $\Delta s$ because the former is a good indicator for the amount of work and memory that is required to find a discrete solution, both for uniform and non-uniform meshes, and the latter is not uniquely defined for non-uniform meshes. Using the sqrtdofs enables us to give a unified analysis for both the uniform and nonuniform schemes.

As noted above we are interested in the rate of convergence of the observed eigenvalue of the discrete system. Let $q_{h}$ be the discrete solution and $\alpha_{\mathrm{L}}\left(q_{h}\right)$ the liquid holdup of the discrete solution. For DG the observed eigenvalue $\omega_{h}$ can be computed by measuring the ratio between the projections of $\alpha_{\mathrm{L}}\left(q_{h}\right)$ on the sinus $e^{i k s}$ at time $t$ and 0 :

$\omega_{h}(t)=\frac{i}{t} \ln \left(\frac{\int_{0}^{\frac{2 \pi}{k}} \alpha_{\mathrm{L}}\left(q_{h}(s, t)\right) e^{-i k s} d s}{\int_{0}^{\frac{2 \pi}{k}} \alpha_{\mathrm{L}}\left(q_{h}(s, 0)\right) e^{-i k s} d s}\right)$.

We deliberately leave out the projection error, hence the term $q_{h}(s$, 0 ) in the denominator instead of $q_{\text {initial }}(s)$. Similarly for FV the observed eigenvalue $\omega_{h}$ is given by

$\omega_{h}(t)=\frac{i}{t} \ln \left(\frac{\sum_{l=0}^{n-1} \alpha_{\mathrm{L}}\left(q_{h}\left(s_{l}, t\right)\right) e^{-i s_{l}} d s}{\sum_{l=0}^{n-1} \alpha_{\mathrm{L}}\left(q_{h}\left(s_{l}, 0\right)\right) e^{-i k s_{l}} d s}\right)$,

where $n$ is the number of cells.

Fig. 6 shows the relative error of the growth rate (see Definition 2) at $t=1 \mathrm{~s}$. For all DG schemes the growth rate converges with order $-(2 p+1)$ with respect to sqrtdofs, or $2 p+1$ with respect to the element width $\Delta s$. That is significantly faster 


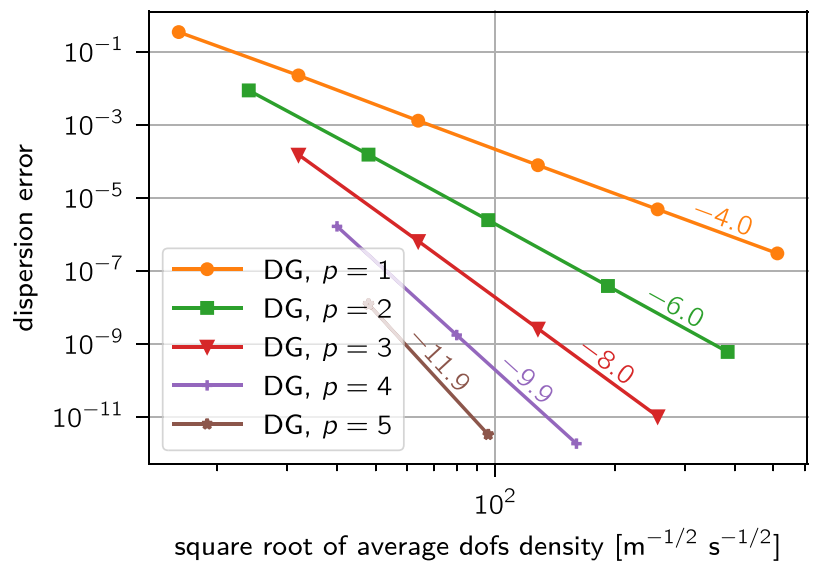

Fig. 7. Convergence of the error of the dispersion at $t=1 \mathrm{~s}$ for the linear, inviscid Kelvin-Helmholtz test-case.

than the rate with which the discrete solution converges. The convergence rate meets the expected convergence rate of the spatial part and the theoretical rate for the temporal part stated in Section 5.1. For the FV scheme, however, the amplitude converges at a rate of -2 with respect to sqrtdofs, which is the same rate as found for the discrete solution. Similarly, Fig. 7 shows the dispersion error (see Defintion 3) at $t=1$. For the DG schemes the dispersion converges even faster, with rate $-(2 p+2)$, which is in accordance with the expected rate of convergence of the spatial part, but it is better than the theoretical rate of convergence for the temporal part. Regarding the growth rate and dispersion the superconverging DG scheme outperforms the FV. Next, we add viscosity to the model and reiterate the above convergence results. The gas dynamic viscosity is set to $\mu_{\mathrm{G}}=1.8 \cdot 10^{-5} \mathrm{~Pa} \mathrm{~s}$, the liquid dynamic viscosity to $\mu_{\mathrm{L}}=8.9 \cdot 10^{-4}$ Pa $\mathrm{s}$ and the pipe roughness to $\epsilon_{\text {pipe }}=10^{-8}$. The turbulent wall friction and interfacial stress is represented by the model of Taitel and Dukler as was described in Section 2. We set the reference state to

$q_{\mathrm{ref}}=\left[\begin{array}{c}p_{\text {int }} \\ h_{\text {int }} \\ u_{\mathrm{L}} \\ u_{\mathrm{G}}\end{array}\right]=\left[\begin{array}{c}10^{5} \mathrm{~Pa} \\ 0 \mathrm{~m} \\ 1 \mathrm{~m} \mathrm{~s}^{-1} \\ 13.978 \ldots \mathrm{m} \mathrm{s}^{-1}\end{array}\right]$,

and add the following artificial body force to the right hand side of both phase momentum Eq. (2)

(76.396...) $A_{\beta}$.

The extra body force makes sure that $q_{\text {ref }}$ is an equilibrium solution of the model. Both the gas velocity and artificial body force coefficient are obtained by numerically solving for the equilibrium condition: zero net momentum source per phase.

Again, we use initial condition (83) with $\omega$ and $r$ equal to the third (algebraically) eigenvalue and eigenvector, and with the amplitude $c$ such that the amplitude of the liquid holdup perturbation is $10^{-10}$ for DG and $10^{-6}$ for FV. The third eigenvalue is given by

$\omega=8.457 \ldots \cdot 10^{0}-3.605 i \cdot 10^{-1}$.

and the third eigenvector by

$r=\left[\begin{array}{c}9.496 \ldots \cdot 10^{-1}+3.062 \ldots i \cdot 10^{-1} \\ -1.604 \ldots \cdot 10^{-4}-2.132 \ldots i \cdot 10^{-5} \\ -1.852 \ldots \cdot 10^{-3}+5.960 \ldots i \cdot 10^{-5} \\ -6.622 \ldots \cdot 10^{-2}-9.132 \ldots i \cdot 10^{-3}\end{array}\right]$

The eigenvalue has a negative imaginary part, hence the initial perturbation will grow in time.

Fig. 8 shows the relative error of the liquid holdup with respect to the exact solution of the linearised model (84). The re-

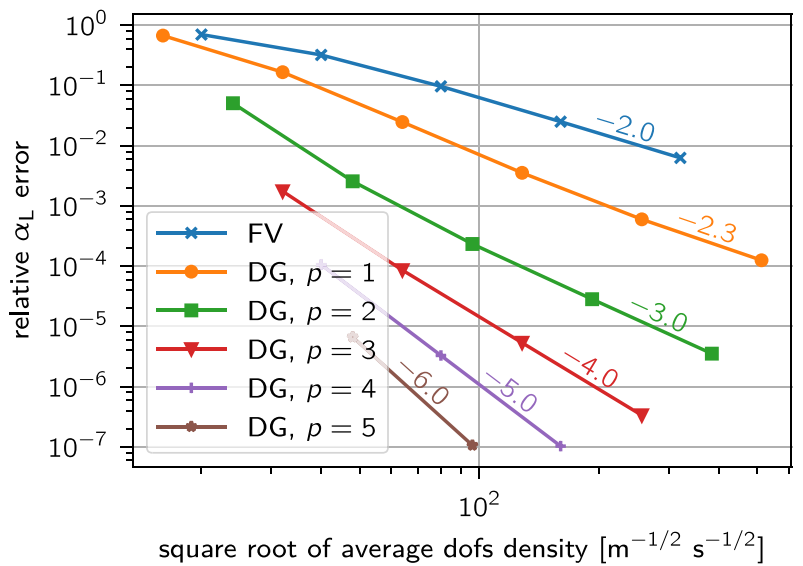

Fig. 8. Convergence of the relative error of the liquid holdup for the linear, viscous Kelvin-Helmholtz test-case.

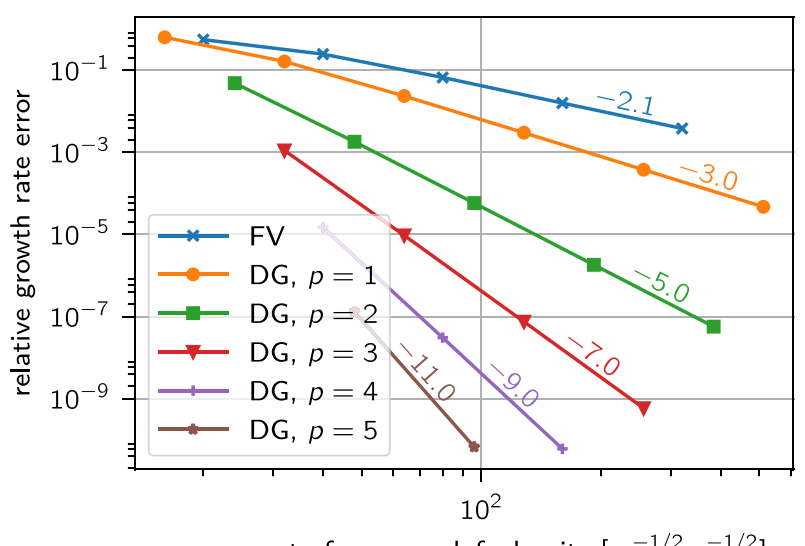

square root of average dofs density $\left[\mathrm{m}^{-1 / 2} \mathrm{~s}^{-1 / 2}\right]$

Fig. 9. Convergence of the relative amplitude error of the liquid holdup for the linear, viscous Kelvin-Helmholtz test-case.

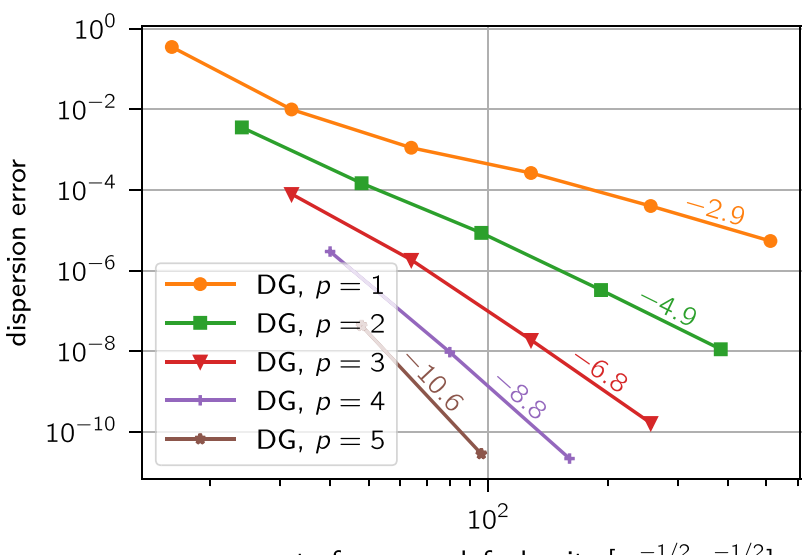

square root of average dofs density $\left[\mathrm{m}^{-1 / 2} \mathrm{~s}^{-1 / 2}\right]$

Fig. 10. Convergence of the phase shift error of the liquid holdup for the linear, viscous Kelvin-Helmholtz test-case.

sults are similar to the inviscid case. For the DG schemes the liquid holdup converges with rate $-(p+1)$ in terms of sqrtdofs. For the FV scheme the rate of convergence is -2 . Also the results for the growth rate and dispersion errors, shown in Figs. 9 and 10, are similar to the inviscid case. The convergence of the dispersion error is now on par with the growth rate error and corresponds to the theoretical analysis of Section 5.1. We proceed with the same viscous model, but we increase the amplitude $c$ of the initial perturbation such that the amplitude of the liquid holdup per- 


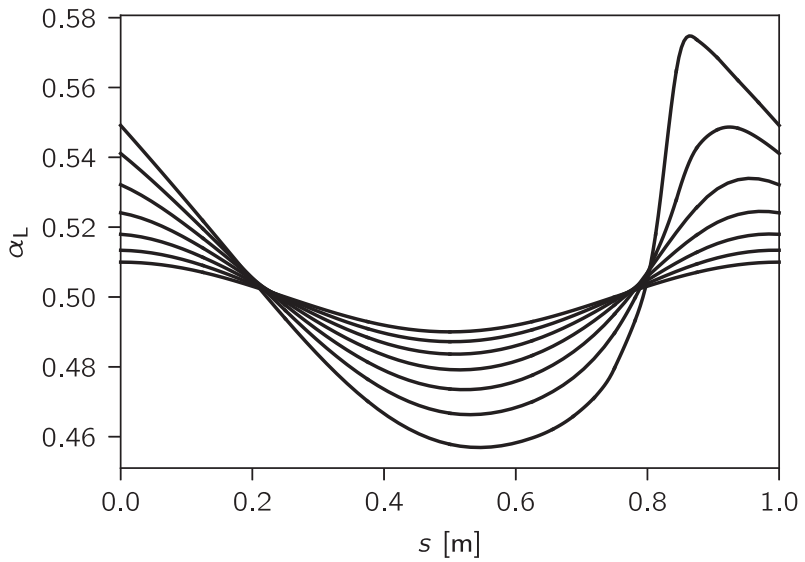

Fig. 11. Liquid holdup part of the discrete solution of the non-linear, viscous Kelvin-Helmholtz test case at time steps $t=n k / \omega, n \in\{0,1, \ldots, 6\}$.

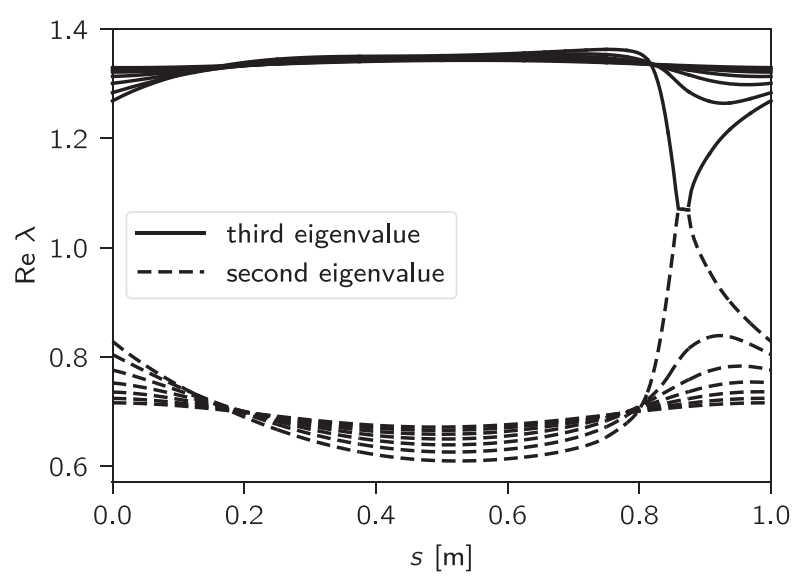

Fig. 12. Second and third eigenvalue of the linearised discrete system of the nonlinear, viscous Kelvin-Helmholtz test case at time steps $t=n k / \omega, n \in\{0,1, \ldots, 6\}$.

turbation is $10^{-2}$. The initial perturbation is now so large that the non-linearity of the model becomes significant. The effect of the non-linearity is visible in Fig. 11, which shows the liquid holdup of a discrete solution on part of the spatial domain at time steps $n k / \omega, n \in\{0,1, \ldots, 6\}$, and in Fig. 13, which shows the amplitude of the perturbation in time. The sinusoidal perturbation of the liquid holdup grows in time and develops a shock. The third eigenvalue, shown in Fig. 12 in the upper half, confirms this: there is a very rapid drop with respect to positive $s$. The second eigenvalue, shown in the lower half of the same figure, grows towards the third eigenvalue. At the last time step displayed the eigenvalues 'touch' each other and form a pair of complex eigenvalues, which marks the end of the well-posedness of the model (see Section 5).

\subsection{Convergence analysis using IFP test case}

We continue with the IFP test case, proposed by the French Petroleum Institute and described by Omgba-Essama [39]. A $10 \mathrm{~km}$ long pipe with a diameter of $0.146 \mathrm{~m}$ is fed at the left side with liquid and gas at constant mass flow rates 20 and $0.2 \mathrm{~kg} \mathrm{~s}^{-1}$, respectively. At the other side the pipe is open at a pressure of $10^{6} \mathrm{~Pa}$. At $t=0 \mathrm{~s}$ the flow is in steady state. Between $t=0$ and $10 \mathrm{~s}$ the gas mass flow rate at the left side changes linearly in time from 0.2 to $0.4 \mathrm{~kg} \mathrm{~s}^{-1}$. In summary, at the left boundary we have

$A_{\beta} \rho_{\beta} u_{\beta}= \begin{cases}20 \mathrm{~kg} \mathrm{~s}^{-1} & \text { if } \beta=\mathrm{L}, \\ 0.2 \mathrm{~kg} \mathrm{~s}^{-1} & \text { if } \beta=\mathrm{G},\end{cases}$

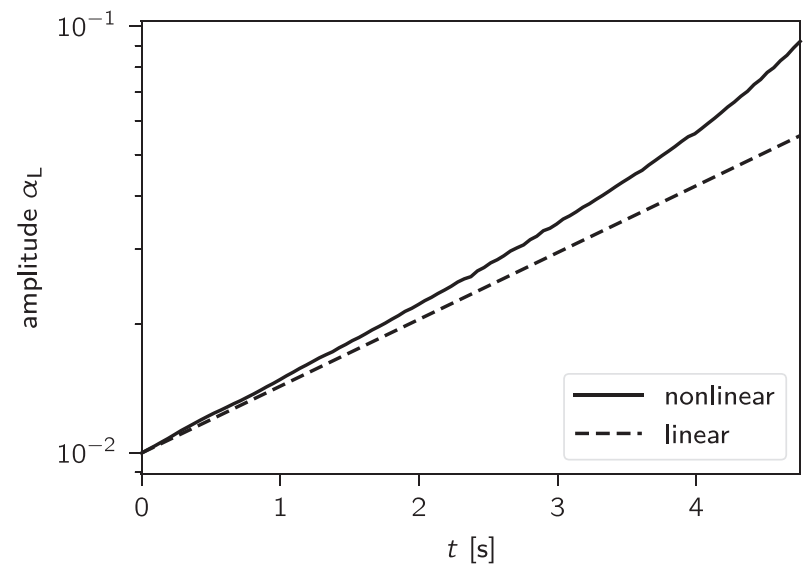

Fig. 13. Evolution of the growth of the discrete liquid holdup of the non-linear and linear, viscous Kelvin-Helmholtz test case.

and at the right boundary

$p=10^{6} \mathrm{~Pa}$.

We use the following equation of state for the gas phase,

$\rho_{\mathrm{G}}=1.26 \frac{p}{10^{5}} \mathrm{~kg} \mathrm{~m}^{-3}$,

and an incompressible water phase with density $\rho_{\mathrm{L}}=$ $1003 \mathrm{~kg} \mathrm{~m}^{-3}$. The equations of state differ from the original problem definition.

The rapid change in the inlet mass flow rate generates a wave that travels to the other side of the domain. The wave consists of a transition in the liquid holdup over 20 to $30 \mathrm{~m}$ and travels with roughly 2 to $3 \mathrm{~m} \mathrm{~s}^{-1}$ through the pipe. After approximately $4500 \mathrm{~s}$ the wave has exited the pipe and the flow slowly settles to a new steady state.

\subsubsection{Analysis of the uniform DG and FV scheme}

We use the Homogeneous Equilibrium Model and apply the second order Finite Volume (FV) scheme and Discontinuous Galerkin (DG) schemes defined earlier to simulate this test case. The ratio between the spatial and the temporal element size, or point distance for $\mathrm{FV}$, is fixed at $16 / 125 \mathrm{~s} \mathrm{~m}^{-1}$, which yields a Courant number of approximately 10 . This is well beyond any CFL condition for explicit schemes. However, since we are using implicit schemes and, for oil and gas applications, are more interested in the relatively slow transport of mass than the fast pressure waves, this choice is justified. For the DG scheme we use a basis of degree 2 and 4 .

Due to the very rapid transition in the liquid holdup and, consequently, short distance over which the liquid holdup changes compared to the length of the pipe, on coarse, uniform meshes the jump is approximately a contact discontinuity. In the FV scheme a limiter is applied to dampen spurious oscillations emanating from this near-discontinuity and in the DG scheme viscosity is added locally, using the approach described in Section 3.4.

Fig. 14 shows the relative $L_{1}$-error of the liquid holdup at $3600 \mathrm{~s}$ for different discretisation schemes. In absence of an exact solution we use, to compute the $L_{1}$-errors, a reference solution obtained using the DG scheme with a sufficiently fine mesh. The horizontal axis displays the square root of the average number of dofs in space and time, abbreviated as sqrtdofs. For the Finite Volume and Discontinuous Galerkin schemes with uniform meshes the sqrtdofs is inversely proportional to the number of spatial grid points or elements, since the ratio of the time step size and the element width, or point distance for FV, is held constant. Doubling 


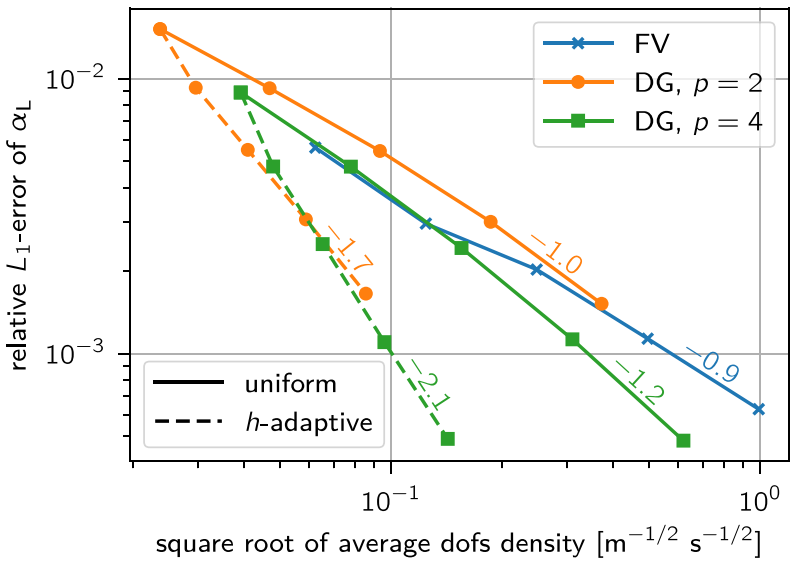

Fig. 14. Convergence of the relative $L_{1}$-error of the liquid holdup in terms of dofs for the IFP test case at $t=3600 \mathrm{~s}$.

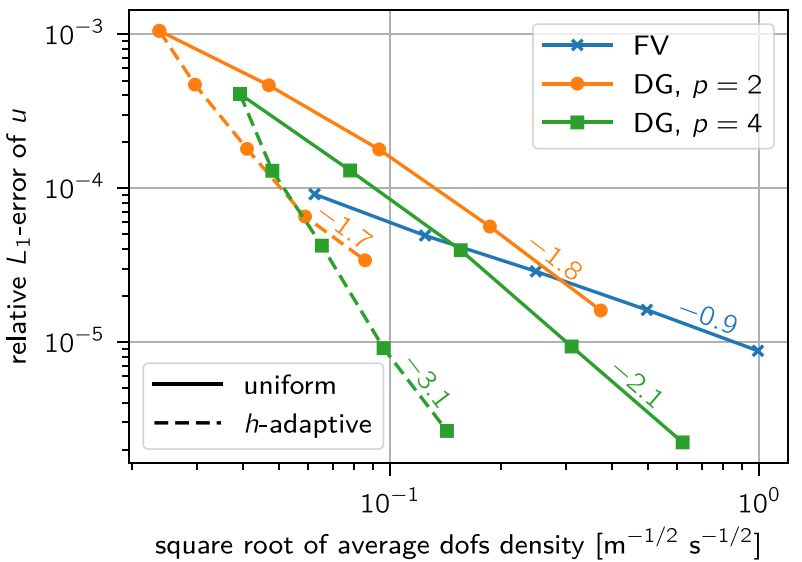

Fig. 15. Convergence of the relative $L_{1}$-error of the velocity in terms of dofs for the IFP test case at $t=3600 \mathrm{~s}$.

the amount of spatial grid points or elements increases the sqrtdofs by a factor two. The FV scheme and DG schemes without local refinement have a comparable performance: all converge roughly with rate minus one in terms of sqrtdofs. Due to the discontinuity in the liquid holdup the theoretical order of convergence in the $L_{1}$-norm is one with respect to the element width, hence minus one in terms of sqrtdofs. The DG scheme with a fourth order basis is slightly more accurate than DG with a second order basis for the same number of dofs and is actually a bit higher than the theoretical limit. This is caused by the viscous limiter being a bit to aggressive on coarse meshes, which adds to the $L_{1}$ error.

Figs. 15 and 16 show the relative $L_{1}$-error of the velocity and pressure at $3600 \mathrm{~s}$ versus sqrtdofs. Contrary to the liquid holdup there is a significant performance difference between the Finite Volume and Discontinuous Galerkin schemes. The FV scheme maintains a convergence rate of roughly minus one, which is similar to the convergence rate for the liquid holdup. The DG schemes, however, have a higher convergence rate ranging from minus one and half to slightly over minus two. Both the velocity and pressure are continuous throughout the simulation, hence the rate of convergence is not theoretically bounded to one in mesh width.

\subsubsection{Analysis of the h-adaptive DG scheme}

To improve the performance we apply local mesh refinement. In the neighbourhood of the discontinuity, as sensed by the smoothness indicator, we repeatedly subdivide elements in two by two parts in space and time until either a predefined maximum num-

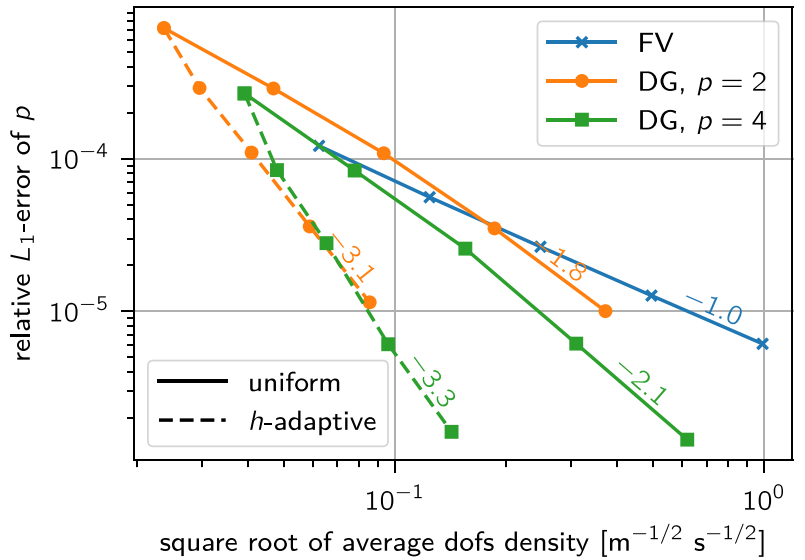

Fig. 16. Convergence of the relative $L_{1}$-error of the pressure in terms of dofs for the IFP test case at $t=3600 \mathrm{~s}$.

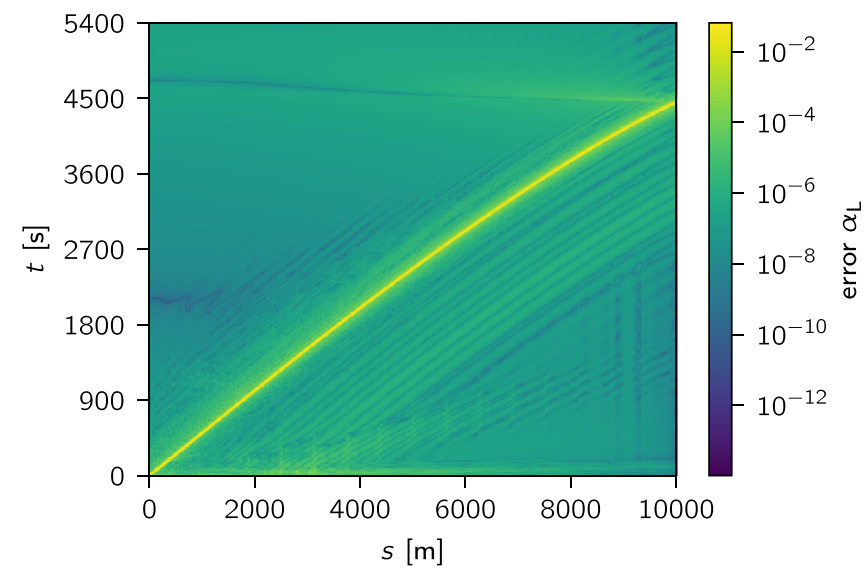

Fig. 17. Pointwise error of the liquid holdup for the IFP test case with a coarse mesh of sixteen spatial elements, a basis of order four and adaptive refinement with a maximum of four levels.

ber of refinements is reached or the smoothness indicator drops below a threshold. The order of the basis functions is unchanged.

Fig. 17 shows the pointwise error of the liquid holdup for a DG scheme with a basis of degree four, sixteen coarse elements in space and a maximum of four levels of refinement - the elements at the finest level coincide with a uniform mesh with 128 spatial elements. The pointwise error is the difference between the solution and the reference solution mentioned above. The pointwise error is inevitably large near the discontinuity, but rapidly decreases away from the discontinuity. Fig. 18 shows the number of refinements: high near the discontinuity and gradually dropping to zero away from the discontinuity, in line with the expectations.

Fig. 19 shows the pointwise error of the liquid holdup at $t=$ $3600 \mathrm{~s}$ for uniform meshes with sixteen and 128 spatial elements and with an $h$-adaptive mesh with sixteen coarse spatial elements and a maximum of three levels of refinement, all with a basis of degree four. The discontinuity is located approximately at $s=$ $7500 \mathrm{~m}$. For all schemes the error is very large near the discontinuity and several orders of magnitude smaller away from the discontinuity. The errors of the fine uniform mesh and the mesh with local refinement, having at its finest level elements of the same size as the fine uniform mesh, are quite similar, which shows that adding more elements in the smooth region does not improve the accuracy.

The convergence results of the $h$-adaptive scheme with an initial mesh of sixteen spatial elements and maximum numbers of refinement ranging from zero to four are displayed in Figs. 14-16 as dashed lines, indicated with ' $h$-adaptive'. Compared to the uniform 


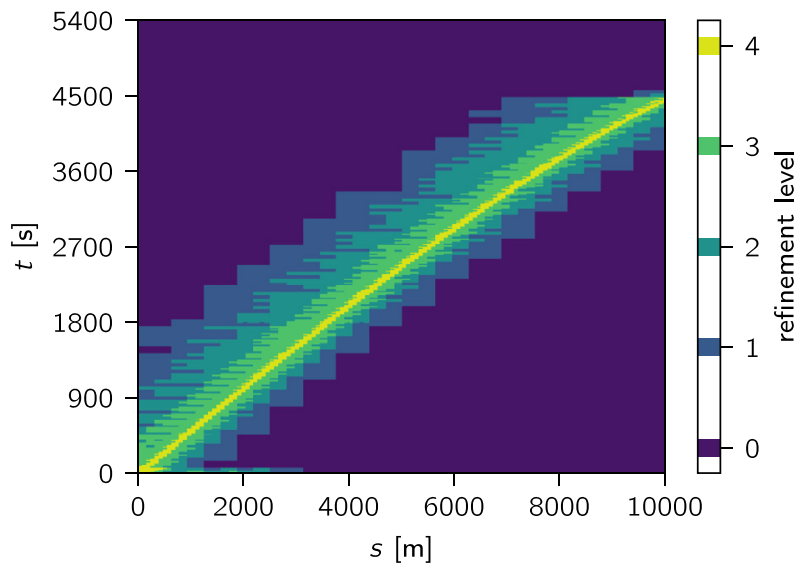

Fig. 18. Local refinement level for the IFP test case with a coarse mesh of sixteen spatial elements, a basis of order four and adaptive refinement with a maximum of four levels.

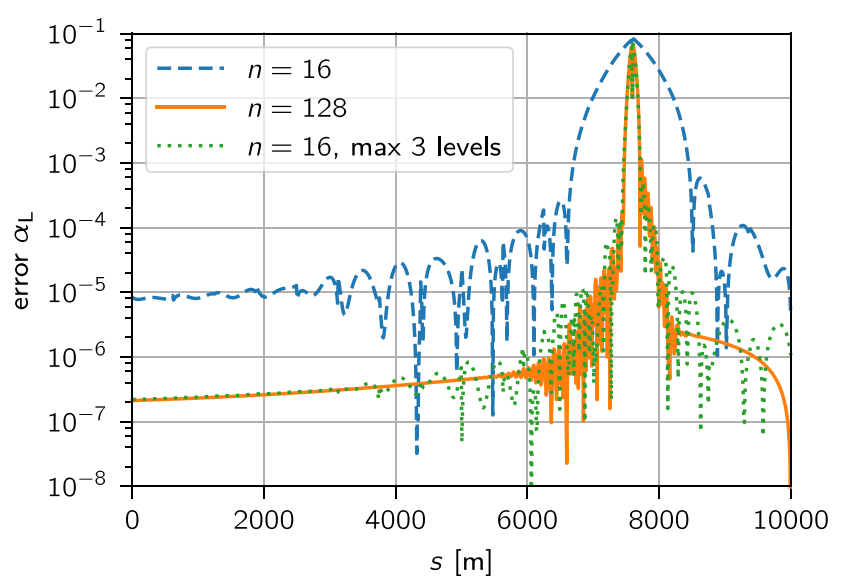

Fig. 19. Comparison of the error of the liquid holdup for the IFP test case at $t=$ $3600 \mathrm{~s}$ for uniform DG schemes with sixteen and 128 spatial elements and the adaptive DG scheme with sixteen spatial coarse elements and a maximum of three levels of refinement, all with a basis of degree four.

DG schemes with bases of equal degree $p$, local refinement significantly improves the performance in terms of sqrtdofs. After four levels of local refinement the rate of convergence with respect to sqrtdofs is up to twice as high as for the uniform schemes. The relative errors of the uniform DG schemes and of the $h$-adaptive DG schemes with at its finest level elements of the same size as the uniform scheme - for example the uniform scheme 64 spatial elements and the $h$-adaptive scheme with sixteen spatial elements and a maximum of two levels of refinement - appear to be roughly the same. This is expected because the error is dominated by the discontinuity and both the uniform and $h$-adaptive schemes have the same mesh width in the neighbourhood of the discontinuity.

The $h$-adaptive scheme requires more work to obtain a solution than the uniform scheme for the same amount of dofs. This is partly because the $h$-adaptive scheme needs to obtain a solution on a series meshes, increasing in level of refinement, before reaching the final mesh. However, when comparing the amount of work for an $h$-adaptive scheme with a uniform scheme that yields the same accuracy we expect the former to be more efficient, given that the difference in amount of dofs is substantial. Fig. 20 shows the performance of the uniform and $h$-adaptive schemes measured in wall clock time. The $h$-adaptive scheme is significantly faster than the uniform scheme for the same accuracy. It must be noted that this depends to some extent on the implementation.

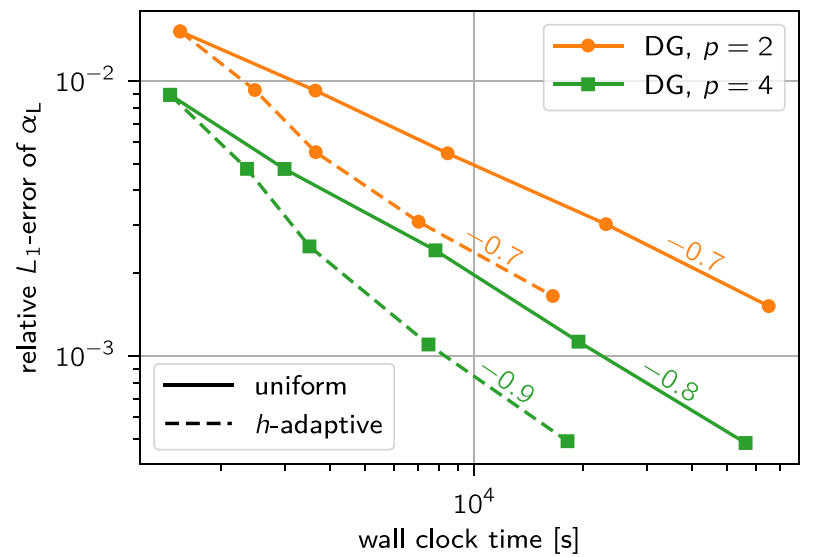

Fig. 20. Convergence of the relative $L_{1}$-error of the liquid holdup in terms of wall clock time for the IFP test case at $t=3600 \mathrm{~s}$.

\section{Conclusions}

We have applied a space-time Discontinuous Galerkin Finite Element Scheme to one-dimensional models for multiphase flow in pipelines and compared the performance with a second order $\mathrm{Fi}$ nite Volume scheme. The solutions of the DGFEM scheme converge in the $L_{2}$-norm with the theoretically expected rate of convergence, which is order $p+1$ in terms of element width, where $p$ is the order of the basis functions. The second order FV scheme shows the expected second order convergence. A linear stability analysis shows that the amplitude and phase shift of a sine wave converge with a higher rate in the DG scheme, being order $2 p+1$ in terms of the mesh width, while the second order FV scheme converges only with order 2 . This shows that a second order DGFEM scheme is superior to a second order FV scheme, with approximately the same number of dofs.

For problems that develop discontinuities, high-order DGFEM methods suffer from spurious oscillations in the neighbourhood of the discontinuities. To suppress these oscillations we have added an artificial diffusion term to the model. The amount of diffusion is determined by the smoothness of the solution: no diffusion in smooth regions and enough diffusion in irregular regions. Because the diffusion term is PDE-based, no special treatment is required on unstructured meshes.

To increase the efficiency of the DGFEM scheme, we have applied local refinement in both space and time. In case of discontinuities, there is a maximum rate of convergence of one with respect to the uniform mesh width. By refining the mesh only in the neighbourhood of discontinuities this limit is surpassed on the global scale. While the refinement scheme requires per time slab solving the discrete system for each refinement level (including the uniform initial mesh), we have shown that the calculation time for the DGFEM scheme with local refinement is shorter compared to the uniform scheme with elements of equal size as the finest elements in the adaptive mesh, while maintaining the same accuracy.

\section{Acknowledgement}

The support of Shell Projects \& Technology in financing the work by the first author is greatly acknowledged.

\section{References}

[1] Stewart BH, Wendroff B. Two-phase flow: models and methods. J Comput Phys 1984;56(3):363-409. doi:10.1016/0021-9991(84)90103-7.

[2] Ishii M, Hibiki T. Thermo-Fluid dynamics of two-phase flow. Springer New York; 2011. doi:10.1007/978-1-4419-7985-8. 
[3] Issa RI, Kempf MHW. Simulation of slug flow in horizontal and nearly horizontal pipes with the two-fluid model. Int J Multiph Flow 2003;29(1):69-95. doi:10.1016/S0301-9322(02)00127-1.

[4] Bendiksen KH, Malnes D, Moe R, Nuland S. The dynamic two-fluid model OLGA: theory and application. Tech. Rep. SPE/19451. Institutt for Energiteknikk; 1991

[5] Evje S, Flåtten T. Hybrid flux-splitting schemes for a common two-fluid model. J Comput Phys 2003;192(1):175-210. doi:10.1016/j.jcp.2003.07.001.

[6] Liao J, Mei R, Klausner J. A study on the numerical stability of the two-fluid model near ill-posedness. Int J Multiph Flow 2008;34(11):1067-87. doi:10. 1016/j.jimultiphaseflow.2008.02.010.

[7] Holmås H. Numerical simulation of waves in two-phase pipe flow using $1 \mathrm{~d}$ two-fluid models. Ph.D. Thesis, University of Oslo; 2008.

[8] Fullmer WD, Ransom VH, Lopez de Bertodano MA. Linear and nonlinear analysis of an unstable, but well-posed, one-dimensional two-fluid model for twophase flow based on the inviscid kelvinhelmholtz instability. Nucl Eng Des 2014;268:173-84. doi:10.1016/j.nucengdes.2013.04.043.

[9] Fullmer WD, Lopez de Bertodano MA, Zhang X. Verification of a higher-order finite difference scheme for the one-dimensional two-fluid model. J Comput Multiph Flows 2013;5(2):139-56. doi:10.1260/1757-482X.5.2.139.

[10] Bonizzi M, Issa RI. On the simulation of three-phase slug flow in nearly horizontal pipes using the multi-fluid model. Int J Multiph Flow 2003;29(11):171947. doi:10.1016/j.ijmultiphaseflow.2003.09.002.

[11] Holmås $\mathrm{H}$, Clamond D, Langtangen HP. A pseudospectral fourier method for a 1d incompressible two-fluid model. Int J Numer Meth Fluids 2008;58(6):63958. doi:10.1002/fld.1772.

[12] Üngör A, Sheffer A. Tent-pitcher: a meshing algorithm for space-time discontinuous Galerkin methods. In: Proceedings of the 9th international meshing roundtable; 2000. https://www.cise.ufl.edu/ ungor/abstracts/imr00.html.

[13] Abedi R, Hawker MA, Haber RB, Matouš K. An adaptive spacetime discontinuous galerkin method for cohesive models of elastodynamic fracture. Int J Numer Meth Eng 2010;81(10):1207-41. doi:10.1002/nme.2723.

[14] Gassner G, Staudenmaier M, Hindenlang F, Atak M, Munz C-D. A spacetime adaptive discontinuous galerkin scheme. Comput Fluids 2015;117:247-61. doi:10.1016/j.compfluid.2015.05.002.

[15] Sollie WEH, Bokhove O, van der Vegt JJW. Spacetime discontinuous galerkin finite element method for two-fluid flows. J Comput Phys 2011;230(3):789817. doi: j.jcp.2010.10.019.

[16] Fidkowski KJ, Luo Y. Output-based space-time mesh adaptation for the compressible navier-stokes equations. J Comput Phys 2011;230(14):5753-73. doi:10.1016/j.jcp.2011.03.059.

[17] Persson P.O., Peraire J. Sub-cell shock capturing for discontinuous galerkin methods. In: Proceedings of the 44th AIAA aerospace sciences meeting and exhibit. American Institute of Aeronautics and Astronautics; doi:10.2514/6. 2006-112.

[18] Barter GE, Darmofal DL. Shock capturing with PDE-based artificial viscosity for DGFEM: part i. formulation. J Comput Phys 2010;229(5):1810-27. doi:10.1016/ j.jcp.2009.11.010

[19] van Zwieten JSB, Sanderse B, Hendrix MHW, Vuik C, Henkes RAWM. Efficient simulation of one-dimensional two-phase flow with a new high-order discontinuous galerkin method. Tech. Rep. 15-07. Delft Institute of Applied Mathematics; 2015.

[20] Taitel Y, Dukler AE. A model for predicting flow regime transitions in horizontal and near horizontal gas-liquid flow. AIChE J 1976;22(1):47-55. doi:10.1002/ aic.690220105.
[21] Vol'pert AI. The spaces $b v$ and quasilinear equations. Math USSR Sbornik 1967;2(2):225-67. doi:10.1070/sm1967v002n02abeh002340.

[22] Dal Maso G, LeFloch PG, Murat F. Definition and weak stability of nonconservative products. J Math Pures Appl 1995;74(6):483-548.

[23] Chalmers N, Lorin E. Approximation of nonconservative hyperbolic systems based on different nonconservative product definitions. Can Appl Math Q 2009;17(3):447-85.

[24] Castro MJ, Pardo A, Parés C, Toro EF. On some fast well-balanced first order solvers for nonconservative systems. Math Comp 2010;79(271):1427-72. doi:10.1090/S0025-5718-09-02317-5.

[25] Toumi I. A weak formulation of Roe's approximate Riemann solver. J Comput Phys 1992;102(2):360-73. doi:10.1016/0021-9991(92)90378-C.

[26] Rhebergen S, Bokhove O, van der Vegt JJW. Discontinuous galerkin finite element methods for hyperbolic nonconservative partial differential equations. J Comput Phys 2008;227(3):1887-922. doi:10.1016/j.jcp.2007.10.007.

[27] Dumbser M, Toro EF. A simple extension of the osher riemann solver to nonconservative hyperbolic systems. J Sci Comput 2011;48(1-3):70-88. doi:10. 1007/s10915-010-9400-3.

[28] Parés C. Numerical methods for nonconservative hyperbolic systems: a theoretical framework. SIAM J Numer Anal 2006;44(1):300-21. doi:10.1137| 050628052.

[29] Baumann CE, Oden JT. A discontinuous hp finite element method for convection-diffusion problems. Comp Meth Appl Mech Eng 1999;175(34):311-41. doi:10.1016/s0045-7825(98)00359-4.

[30] Bassi F, Rebay S. Numerical evaluation of two discontinuous galerkin methods for the compressible navier-stokes equations. Int J Numer Meth Fluids 2002;40(1-2):197-207. doi:10.1002/fld.338.

[31] Bassi F, Rebay S. GMRES Discontinuous galerkin solution of the compressible navier-stokes equations. In: Cockburn B, Karniadakis G, Shu C-W, editors. Discontinuous Galerkin methods. Lecture Notes in Computational Science and Engineering, vol. 11. Springer Berlin Heidelberg; 2000. p. 197-208. doi:10.1007/978-3-642-59721-3_14.

[32] Arnold DN, Brezzi F, Cockburn B, Marini LD. Unified analysis of discontinuous galerkin methods for elliptic problems. SIAM J Numer Anal 2002;39(5):174979. doi:10.1137/S0036142901384162.

[33] Davis TA. Algorithm 832: umfpack v4.3-an unsymmetric-pattern multifrontal method. ACM Trans Math Softw 2004;30(2):196-9. doi:10.1145/992200. 992206.

[34] van Zwieten JSB. joostvanzwieten/sample-code-paper-hstdg2017 v1; 2017. doi:10.5281/zenodo.820673.

[35] Olsen R. Time-dependent boundary conditions for multiphase flow. Ph.D. Thesis, Norwegian University of Science and Technology (NTNU); 2004.

[36] Prosperetti A. Two-fluid modelling and averaged equations. Multiph Sci Technol 2003;15:181-92. doi:10.1615/MultScienTechn.v15.i1-4.160.

[37] Ainsworth M. Dispersive and dissipative behaviour of high order discontinuous galerkin finite element methods. J Comput Phys 2004;198(1):106-30. doi:10. 1016/j.jcp.2004.01.004.

[38] Lesaint P, Raviart PA. On a finite element method for solving the neutron transport equation. In: Boor $C D$, editor. Mathematical aspects of finite elements in partial differential equations. Academic Press, New York; 1974. p. 89-123. doi:10.1016/B978-0-12-208350-1.50008-X.

[39] Omgba-Essama C. Numerical modelling of transient gas-liquid flows (application to stratified slug flow regimes). Ph.D. Thesis, Cranfield University; 2004. 